\title{
Microwave experiments on correlated disorder
}

\author{
Ulrich Kuhl \\ Fachbereich Physik, Philipps-Universität Marburg, Germany \\ LPMC, CNRS UMR 6622, Université de Nice Sophia-Antipolis, France
}




\title{
Microwave experiments
}

\author{
on correlated disorder
}

\author{
Ulrich Kuhl \\ Fachbereich Physik, Philipps-Universität Marburg, Germany \\ LPMC, CNRS UMR 6622, Université de Nice Sophia-Antipolis, France \\ 14. July 2010, Cargèse, Corse, France
}

ulrich.kuhl@unice.fr 


\section{Collaborations}

\& H.-J. Stöckmann

Fachbereich Physik der Philipps-Universität Marburg, D-35032 Marburg, Germany

D. Dietz

Fachbereich Physik der Philipps-Universität Marburg, D-35032 Marburg, Germany

- F. M. Izrailev

Instituto de Física, Universidad Autónoma de Puebla, Puebla 72570, Mexico

- A. A. Krokhin

Department of Physics, University of North Texas, P.O. Box 311427, Denton, Texas 76203, USA

- G. Luna-Acosta

Instituto de Física, Universidad Autónoma de Puebla, Puebla 72570, Mexico

¿ N. M. Makarov

Instituto de Física, Universidad Autónoma de Puebla, Puebla 72570, Mexico 


\section{Contents}

- One dimensional waveguide

๖ Setup

๑ Periodic arrangements

- Hofstadter butterfly

- Correlated disorder

$\downarrow \quad$ Band edge

๑ Enhanced localization

¿ Fabry-Perot bands using positional disorder

¿ Quasi 1D systems

\& Setup

๖ Rectangular waveguide

¿ Correlated disorder

¿ Fabry-Perot
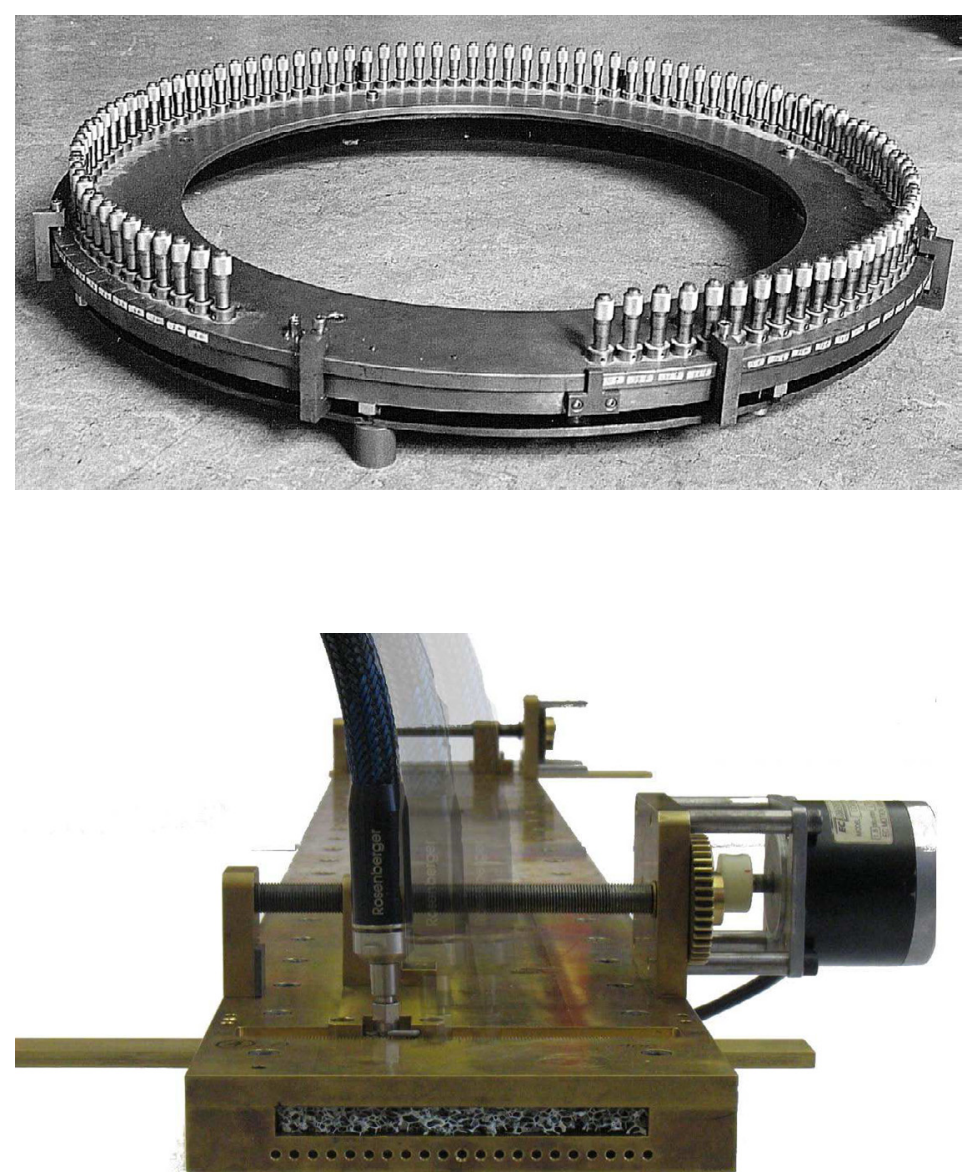

¿ Summary

¿ Publications 


\section{One dimensional Waveguides (1D)}

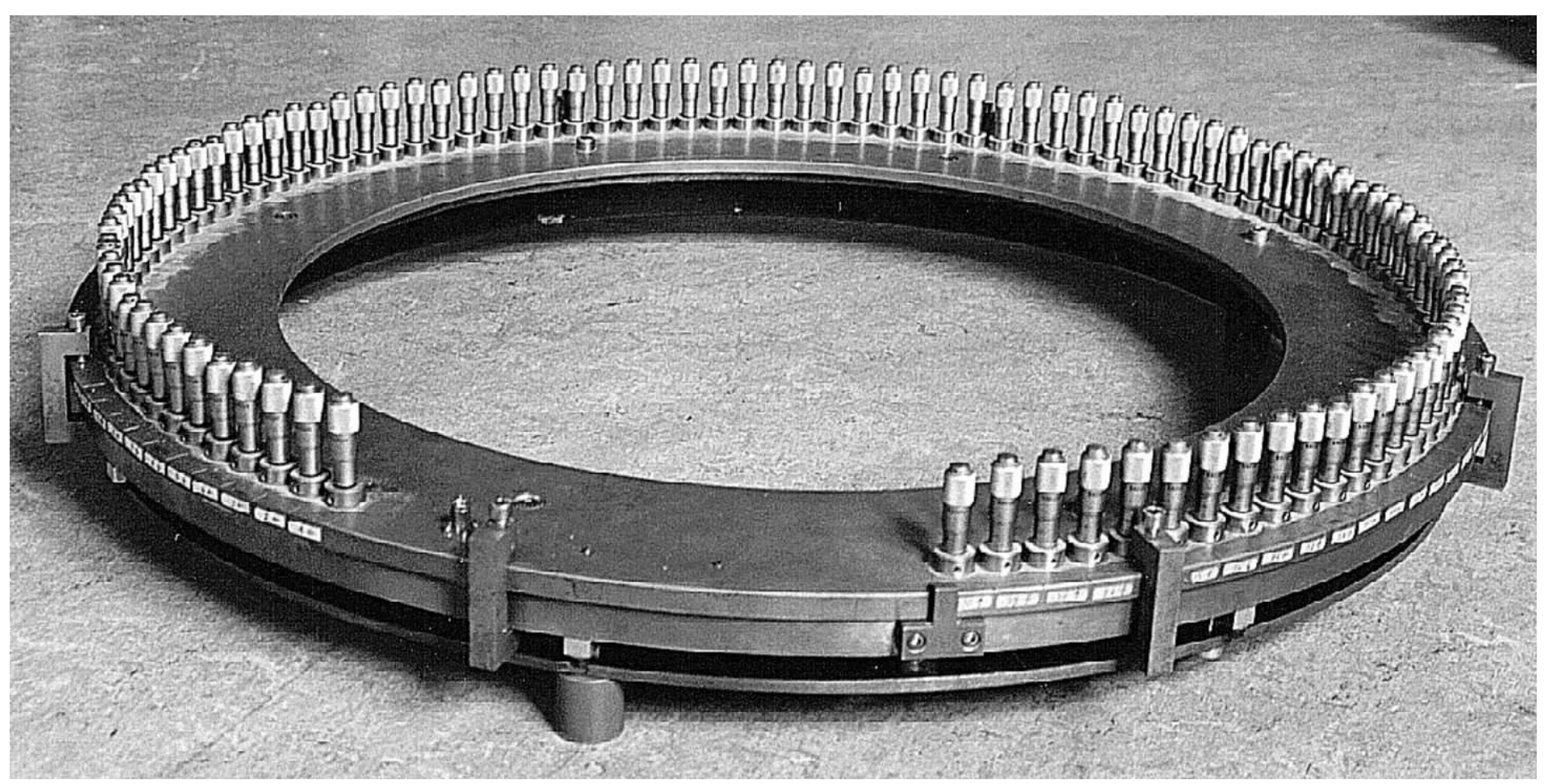




\section{Microwave set-up}
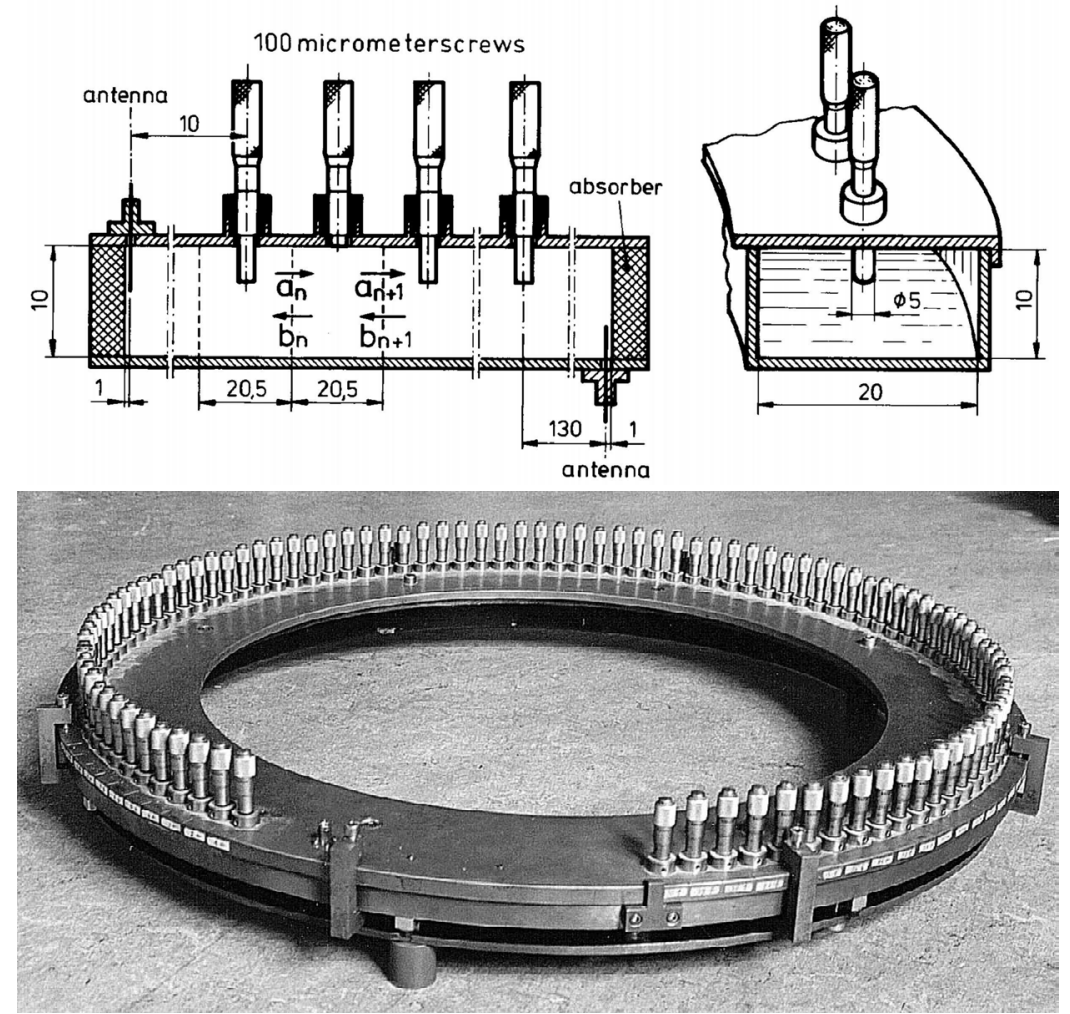

Only one mode propagates in the frequency range:

$$
7.5 \mathrm{GHz} \leq \nu \leq 15 \mathrm{GHz} \text {. }
$$

$\Rightarrow$ wavenumber range $\left(k=\frac{2 \pi}{c} \sqrt{\nu^{2}-\nu_{0}^{2}}\right): \quad 0 \leq k /(\pi / d) \leq 1.8$

The experiment measures the transmission $\propto \frac{1}{\operatorname{Tr}\left(T^{(N)}\right)}$ 


\section{One-dimensional waveguide}

$$
\begin{aligned}
& \begin{array}{l}
a_{n-1} \rightarrow \\
b_{n-1} \leftarrow \\
\leftarrow b_{n} \leftarrow a_{n} \rightarrow \\
\leftarrow b_{n+1}
\end{array} \\
& \longleftarrow d
\end{aligned}
$$

Only one mode propagates $\Rightarrow$ transfer matrix ansatz:

$$
\left(\begin{array}{c}
a_{n+1} \\
b_{n+1}
\end{array}\right)=T_{n}\left(\begin{array}{c}
a_{n} \\
b_{n}
\end{array}\right)
$$

Assumptions:

( $\quad$ time-reversal symmetry $\Rightarrow r_{n}=r_{n}^{*}$ and $t_{n}=t_{n}^{*}$

( no absorption $\Rightarrow\left|r_{n}\right|^{2}+\left|t_{n}\right|^{2}=1$

$$
T_{n}=\left(\begin{array}{cc}
\frac{1}{t_{n}} e^{i k d} & \frac{r_{n}}{t_{n}} e^{-i k d} \\
\frac{r_{n}^{*}}{t_{n}^{*}} e^{i k d} & \frac{1}{t_{n}^{*}} e^{-i k d}
\end{array}\right)
$$

$k d$ : phase shift without scatterer 


\section{Periodic structures}

Every third (a) or every fourth (b) micrometer screw is introduced $3 \mathrm{~mm}$ in the waveguide:

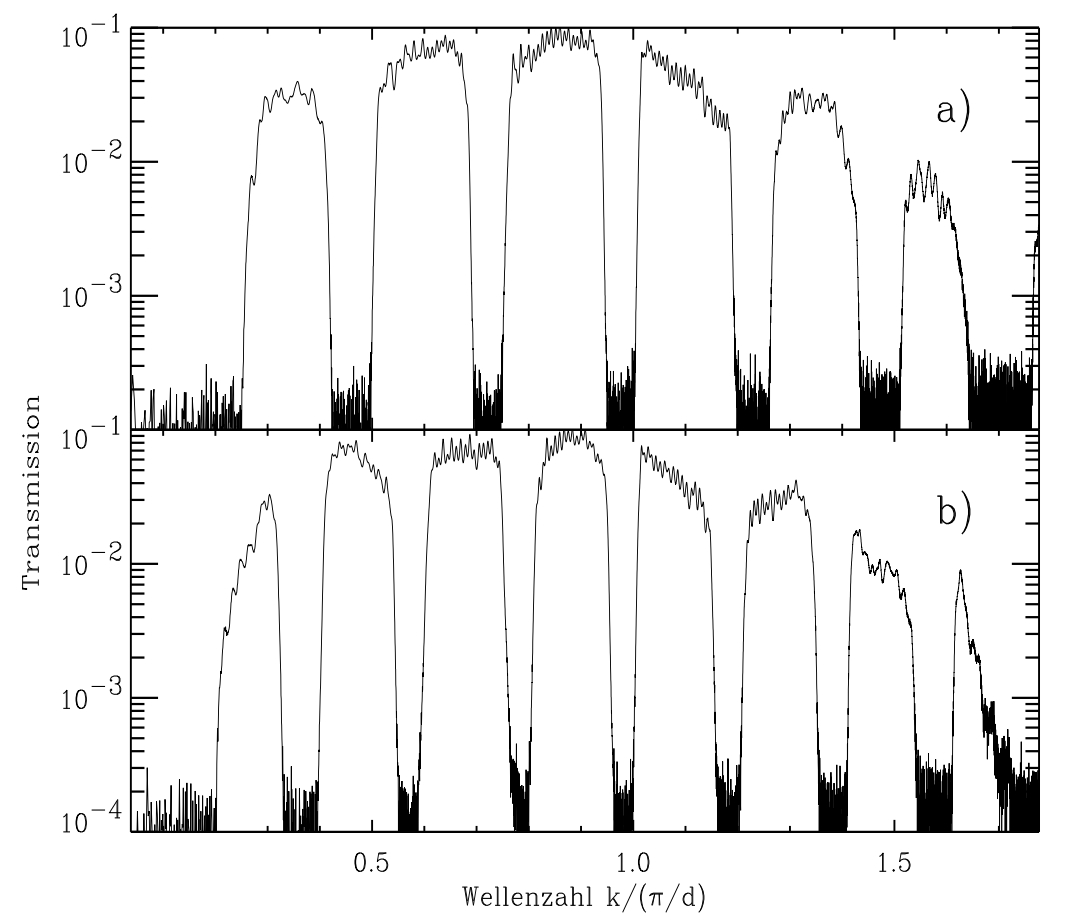

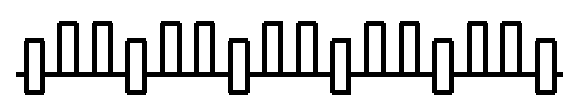

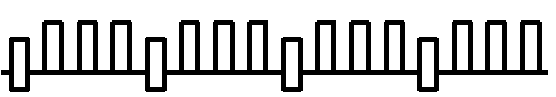




\section{Wavefunctions}

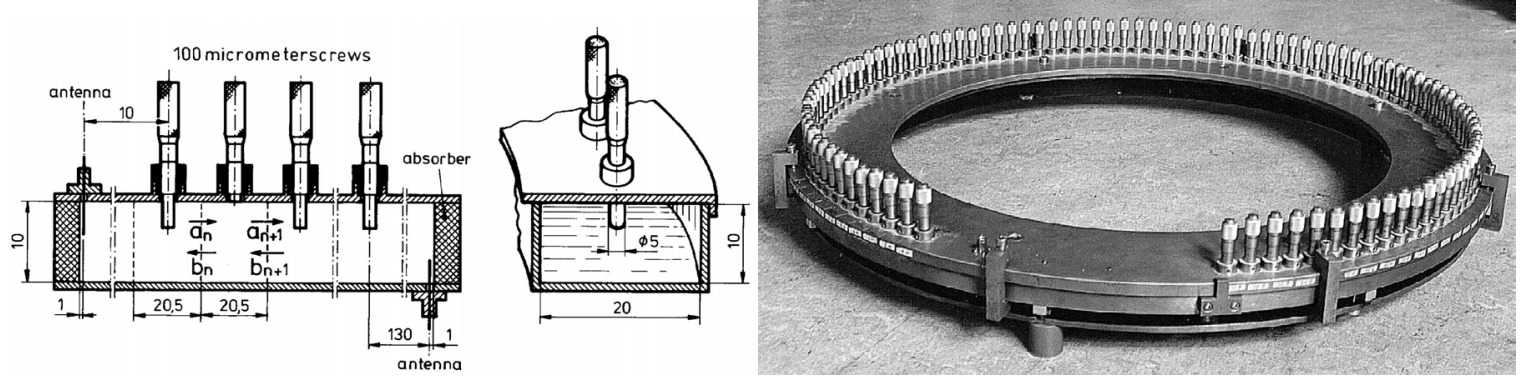

Reflection measurement:

Every fifth scatterer is introduced $3 \mathrm{~mm}$ :

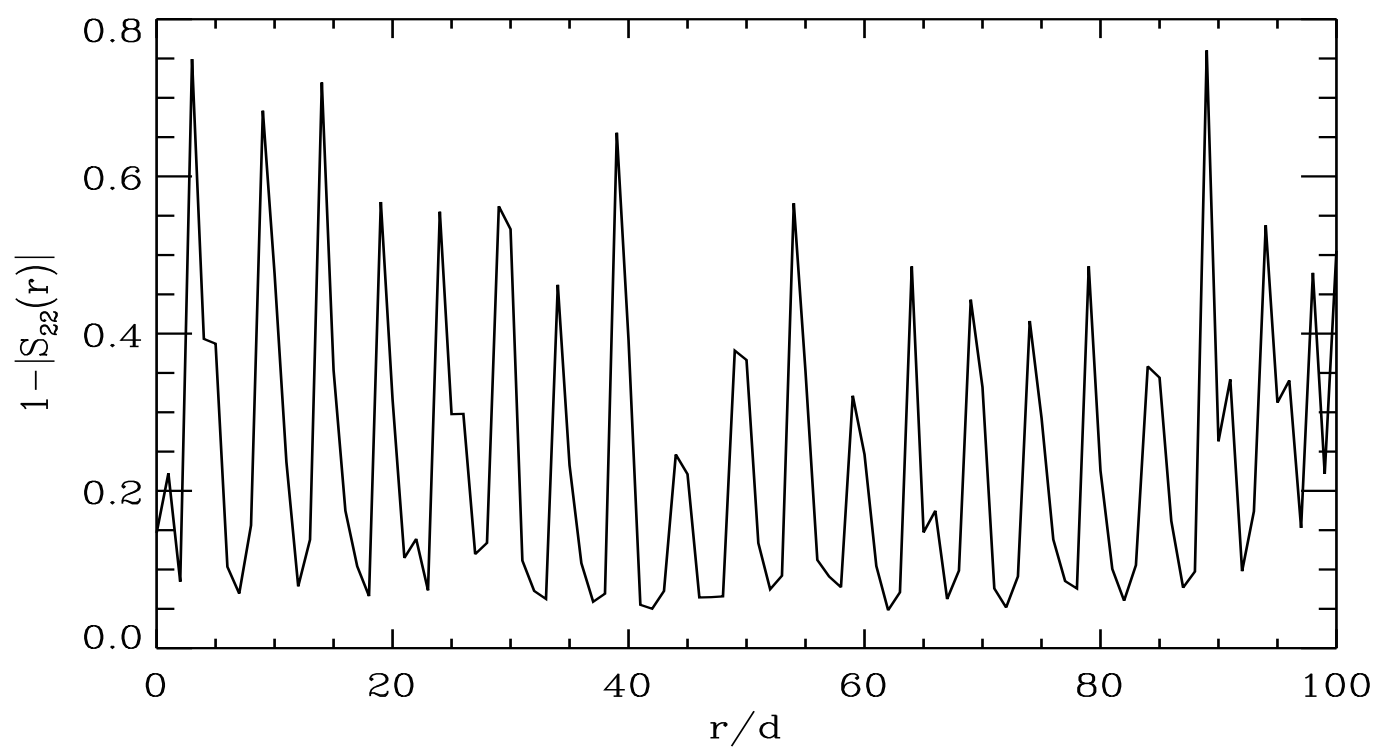

Bloch oscillations are observed. 


\section{Hofstadter butterfly}

Hofstadter calculated the conductance of a twodimensional crystal with a perpendicularly applied magnetic field $B$ (Hofstadter 1976):

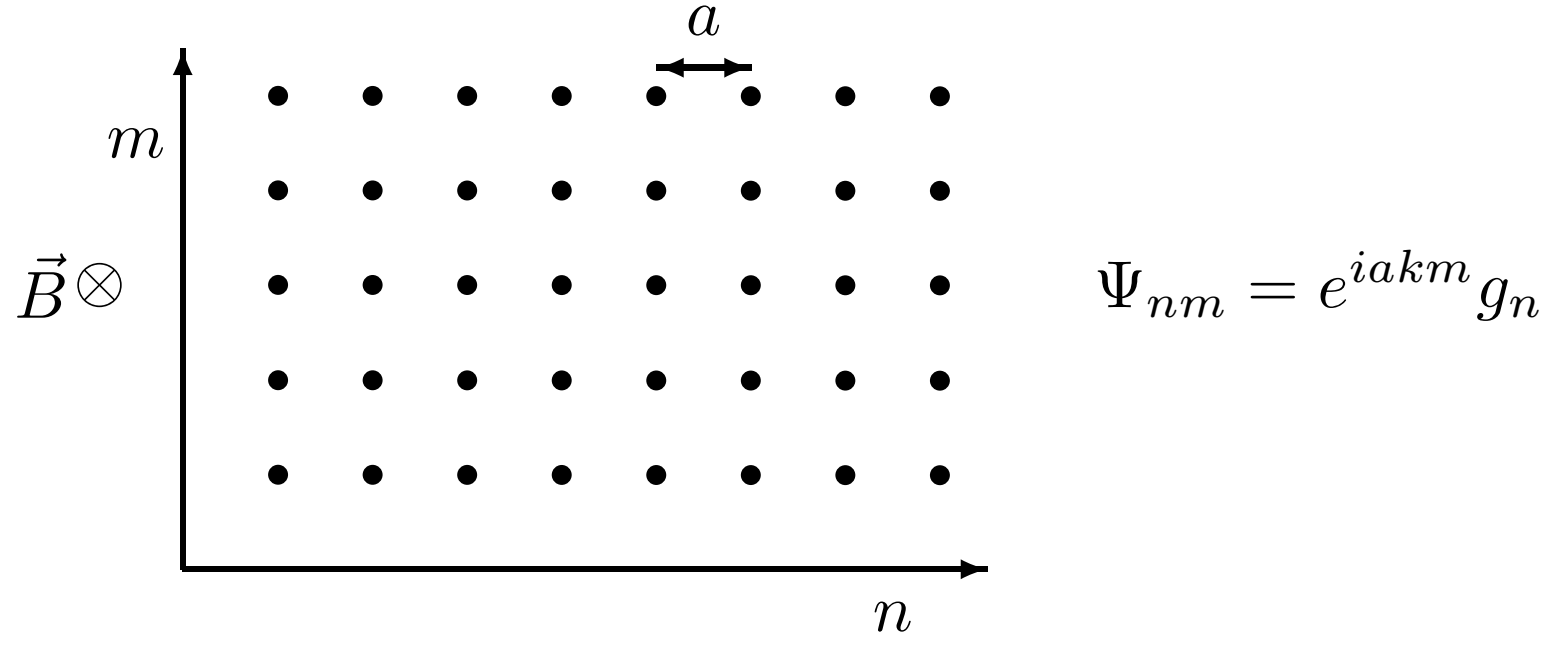

The $g_{n}$ obey the Harper equation:

$$
g_{n+1}+g_{n-1}+2 \cos (2 \pi n \alpha-\nu) g_{n}=E g_{n}
$$

with

$$
\alpha=\frac{a^{2} e B}{h}
$$

$\alpha$ : number of magnetic flux quanta per unit cell 


\section{Hofstadter butterfly}

$\alpha$ rational $\frac{p}{q} \Rightarrow$ Spectrum splits into $q$ subbands.

$\alpha$ irrational $\Rightarrow$ Spectrum forms a Cantor Set

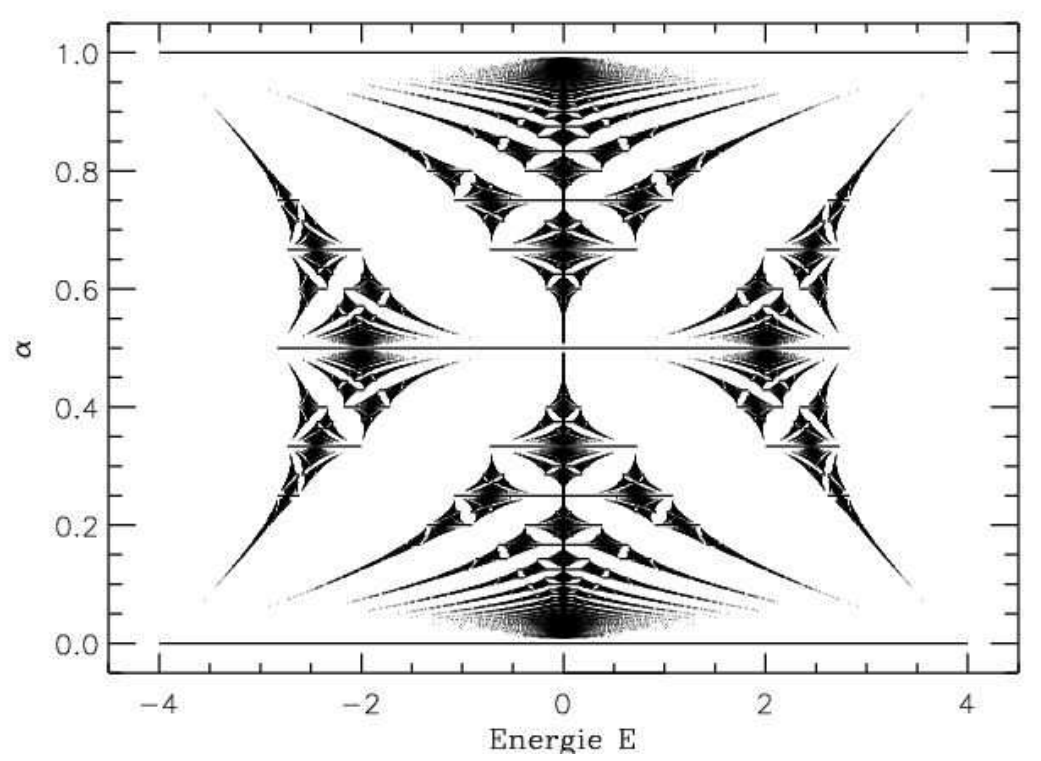

- spectra are self-similar.

- $a$ typically $10^{-10} \mathrm{~m} \Rightarrow B \approx 10^{5} \mathrm{~T}$ needed.

Alternative: superlattices (Schlösser et al. 1996) 


\section{Harper modell vs waveguide}

Harper equation: $g_{n+1}+g_{n-1}+2 \cos (2 \pi n \alpha-\nu) g_{n}=E g_{n}$ too, allows a transfer matrix notation:

$$
\left(\begin{array}{c}
g_{N+1} \\
g_{N}
\end{array}\right)=T^{(N)}\left(\begin{array}{c}
g_{1} \\
g_{0}
\end{array}\right)
$$

with $T^{(N)}=\prod_{n=0}^{N} T_{n}$, where

$$
T_{n}=\left(\begin{array}{cc}
E-2 \cos (2 \pi n \alpha-\nu) & -1 \\
1 & 0
\end{array}\right)
$$

One-dimensional Waveguide:

$$
T_{n}=\left(\begin{array}{cc}
\frac{1}{t_{n}} e^{i k d} & \frac{r_{n}}{t_{n}} e^{-i k d} \\
\frac{r_{n}^{*}}{t_{n}^{*}} e^{i k d} & \frac{1}{t_{n}^{*}} e^{-i k d}
\end{array}\right)
$$

Transmission if:

$$
\lim _{N \rightarrow \infty} \frac{1}{\operatorname{Tr}\left(T^{(N)}\right)} \begin{cases}\neq 0 & \text { allowed bands (Bloch band) } \\ =0 & \text { forbidden bands (band gap) }\end{cases}
$$




\section{Spectra for different $\alpha$}

Micrometer screw $n$ is introduced $3 \mathrm{~mm}$ in the waveguide, if $\cos (2 \pi n \alpha)>0$ :
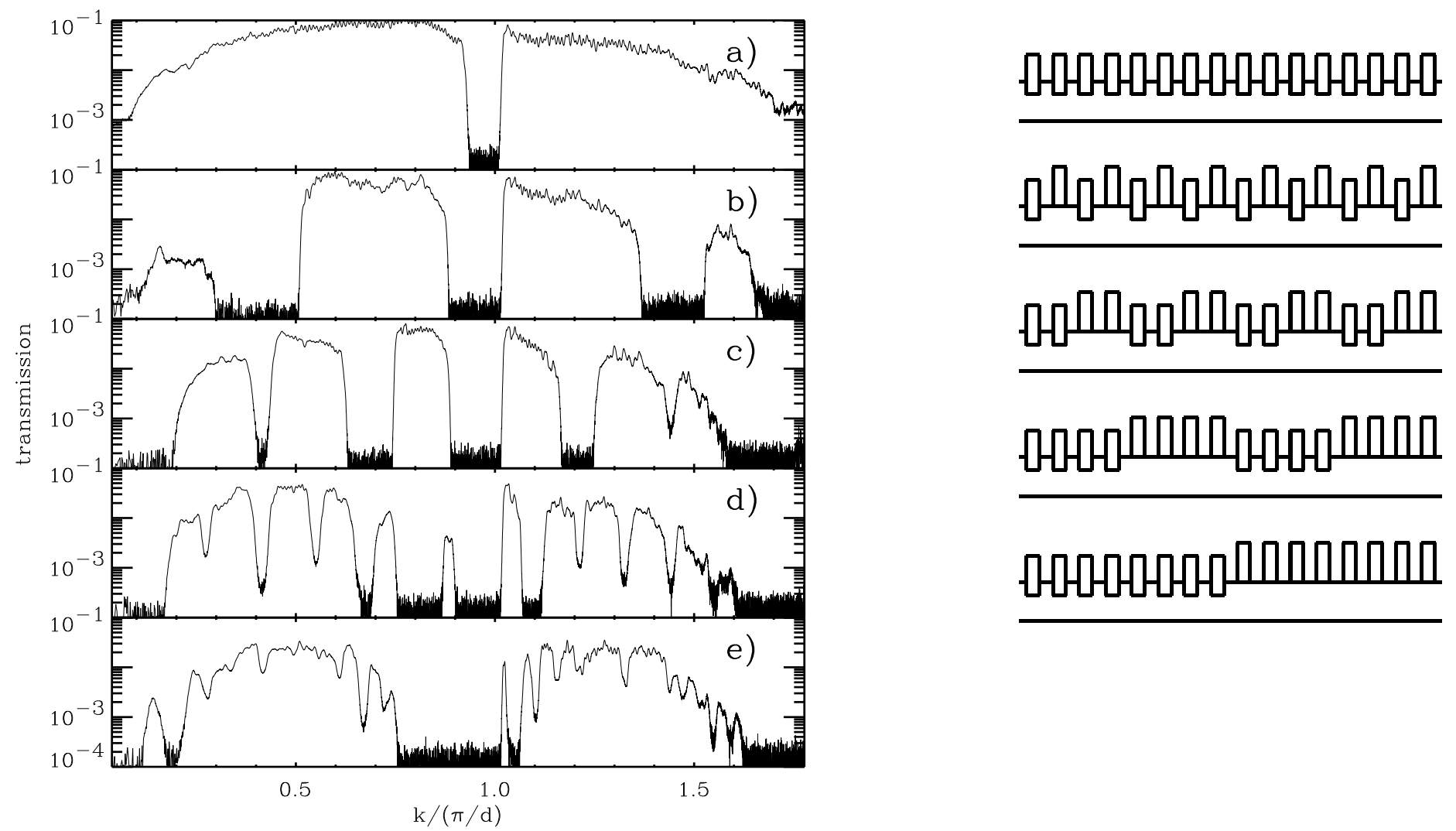

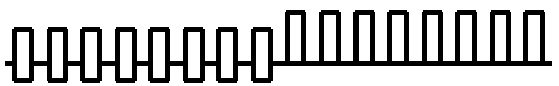

$$
\alpha=1 / 1 \text { (a), } 1 / 2 \text { (b), 1/4 (c), 1/8 (d), 1/16 (e) }
$$

[U.K., H.-J. Stöckmann. PRL 80, 3232 (1998)] 


\section{Microwave Hofstadter butterfly}

Transmission as function of $k$ and $\alpha$ in greyscale printing reproduces the Hofstadter butterfly in every Bloch band:
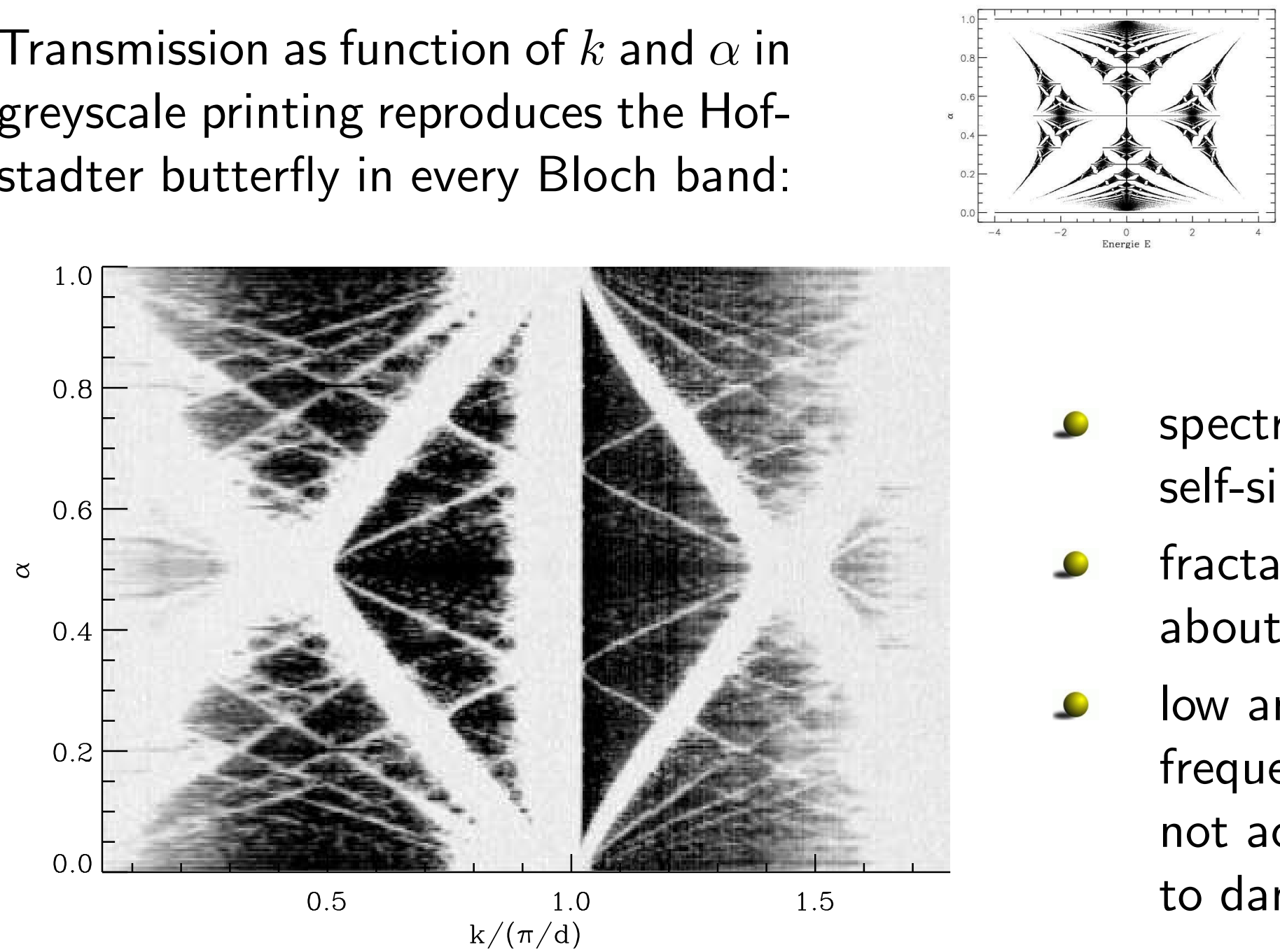

- spectra are self-similar

- fractal depth of about 3 or 4

- low and high frequency range is not accessible due to damping

[U.K., H.-J. Stöckmann. PRL 80, 3232 (1998)] 


\section{Butterfly for different $d$}

Using only every second micrometer screw doubles the number of Bloch bands in the accessible frequency range:

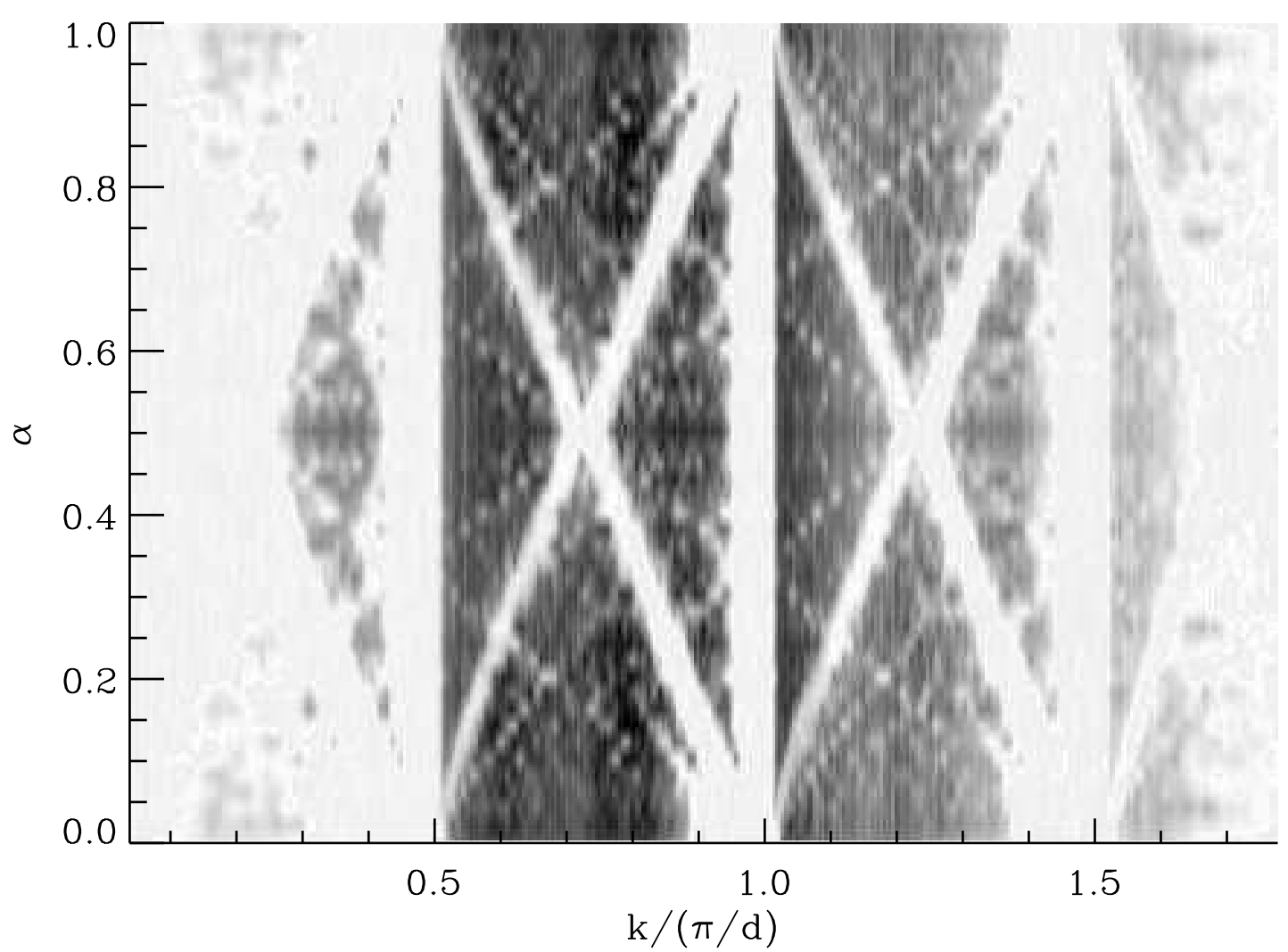

¿ butterflies in second and third band complete

¿ usage of half of the scatterers $\Rightarrow$ smaller fractal depth 


\section{Experiment versus scattering theory}

Calculating the transmission for 100 scatterers in a rectangular scattering arrangement by multiplying

$$
T_{n}=\left(\begin{array}{ll}
\frac{1}{t_{n}} e^{i k d} & \frac{r_{n}}{t_{n}} e^{-i k d} \\
\frac{r_{n}^{*}}{t_{n}^{*}} e^{i k d} & \frac{1}{t_{n}^{*}} e^{-i k d}
\end{array}\right)
$$
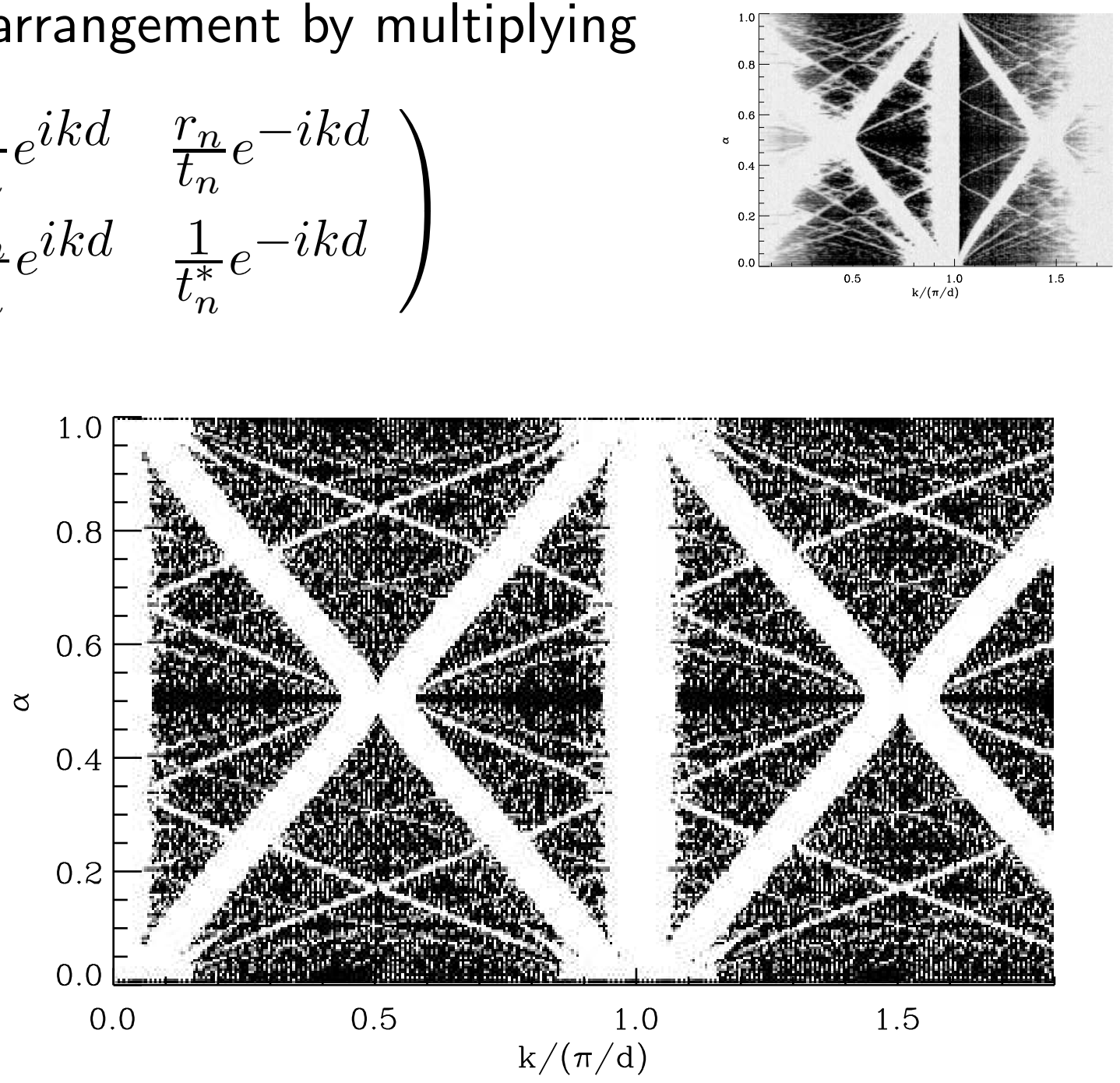


\title{
Correlated disorder
}

\author{
Band edges
}




\section{Correlated disorder (tight binding)}

The tight-binding model

$$
\psi_{n+1}+\psi_{n-1}=\left(E+\epsilon_{n}\right) \psi_{n},
$$

with $\epsilon_{n}$ : site potential,

The inverse localization length within the band $(|E|<2)$ is given

by: $\quad l^{-1}=\frac{\left\langle\epsilon_{n}^{2}\right\rangle \varphi(\mu)}{8 \sin ^{2} \mu}$

$$
\varphi(\mu)=1+2 \sum_{k=1}^{\infty} \xi(k) \cos (2 \mu k), \text { where } E=2 \cos \mu
$$

$\xi(k)$ : two-point correlators of the site potential $\epsilon_{n}$

$$
\xi(k)=\frac{2}{\pi} \int_{0}^{\pi / 2} \varphi(\mu) \cos (2 k \mu) \mathrm{d} \mu
$$

Arbitrary band structures can be generated e.g. via:

$$
\epsilon_{n}=A \sum_{k=-\infty}^{\infty} \xi(k) Z_{n+k}
$$




\section{Correlated disorder (Kronig-Penney)}

Discrete Kronig-Penney model

$$
\psi_{n+1}+\psi_{n-1}=\left[2 \cos (k d)+U_{n} k d \sin (k d)\right] \psi_{n}
$$

Split potential into mean $\epsilon$ and fluctuating $\epsilon_{n}$ parts:

$$
U_{n}=\epsilon+\epsilon_{n} .
$$

Equivalent to tight-binding model with

$$
\begin{aligned}
E & \rightarrow 2 \cos (k d)+k \epsilon \sin (k d) \\
\epsilon_{n} & \rightarrow k \epsilon_{n} \sin (k d): \text { random potential, } d \text { scatterer distance }
\end{aligned}
$$

Localization length

$$
l_{\infty}^{-1}=\frac{\epsilon_{0}^{2}}{8 k^{2}} \frac{\sin (k d)}{\sin ^{2}(\gamma)}, \quad \epsilon_{0}=\left\langle\epsilon_{n}^{2}\right\rangle
$$

with

$$
2 \cos (\gamma)=2 \cos (k d)+\frac{\epsilon_{0}}{k} \sin (k d), \quad E=k^{2}
$$




\section{Band structures}

Transmission through generated correlated disorder arrangement

Numerics:

$$
\begin{aligned}
& N=10^{4} \\
& \left\langle\epsilon_{n}\right\rangle=-0.1 \\
& \sqrt{\left\langle\epsilon_{n}^{2}\right\rangle}=0.1
\end{aligned}
$$

Measurement:

Single $(\mathrm{N}=100)$ and average of 5

Multiplication of 5 measurements $(\mathrm{N}=500)$

[U.K., F. M. Izrailev, A. A. Krokhin, H.-J. Stöckmann. APL 2000]

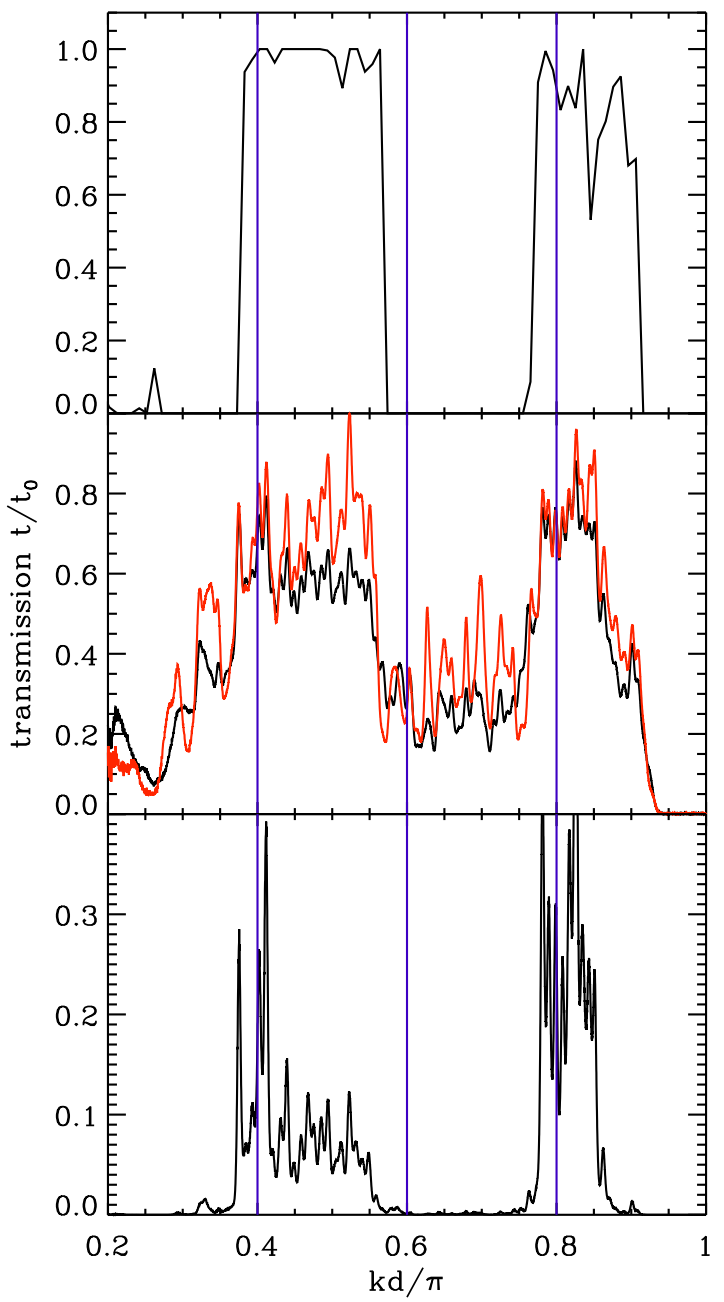




\section{Inverse band structures}

Transmission through generated correlated disorder arrangement

Numerics:

$N=10^{4}$

$\left\langle\epsilon_{n}\right\rangle=-0.1$

$\sqrt{\left\langle\epsilon_{n}^{2}\right\rangle}=0.1$

Measurement:

Single $(\mathrm{N}=100)$ and average of 5

Multiplication of 5 measurements $(\mathrm{N}=500)$

[U.K., F. M. Izrailev, A. A. Krokhin, H.-J. Stöckmann. APL 2000]

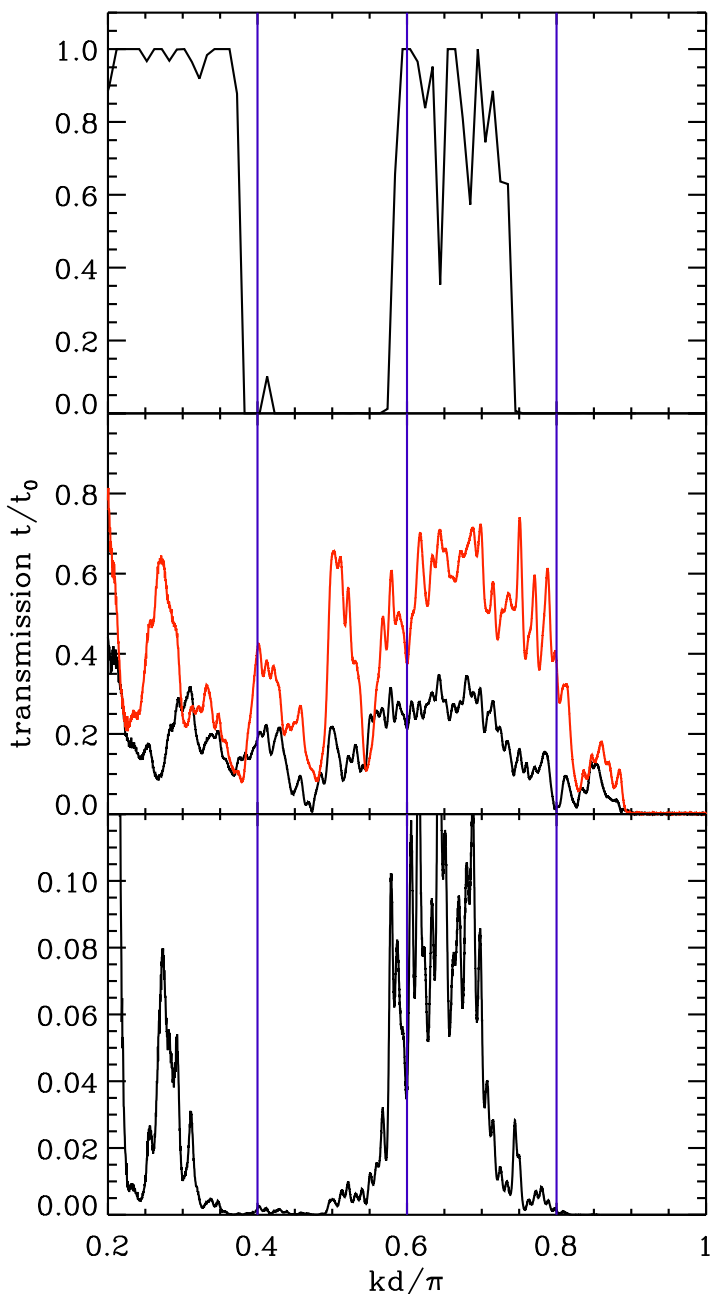




\section{Random structures}

Transmission through a random scattering arrangement:

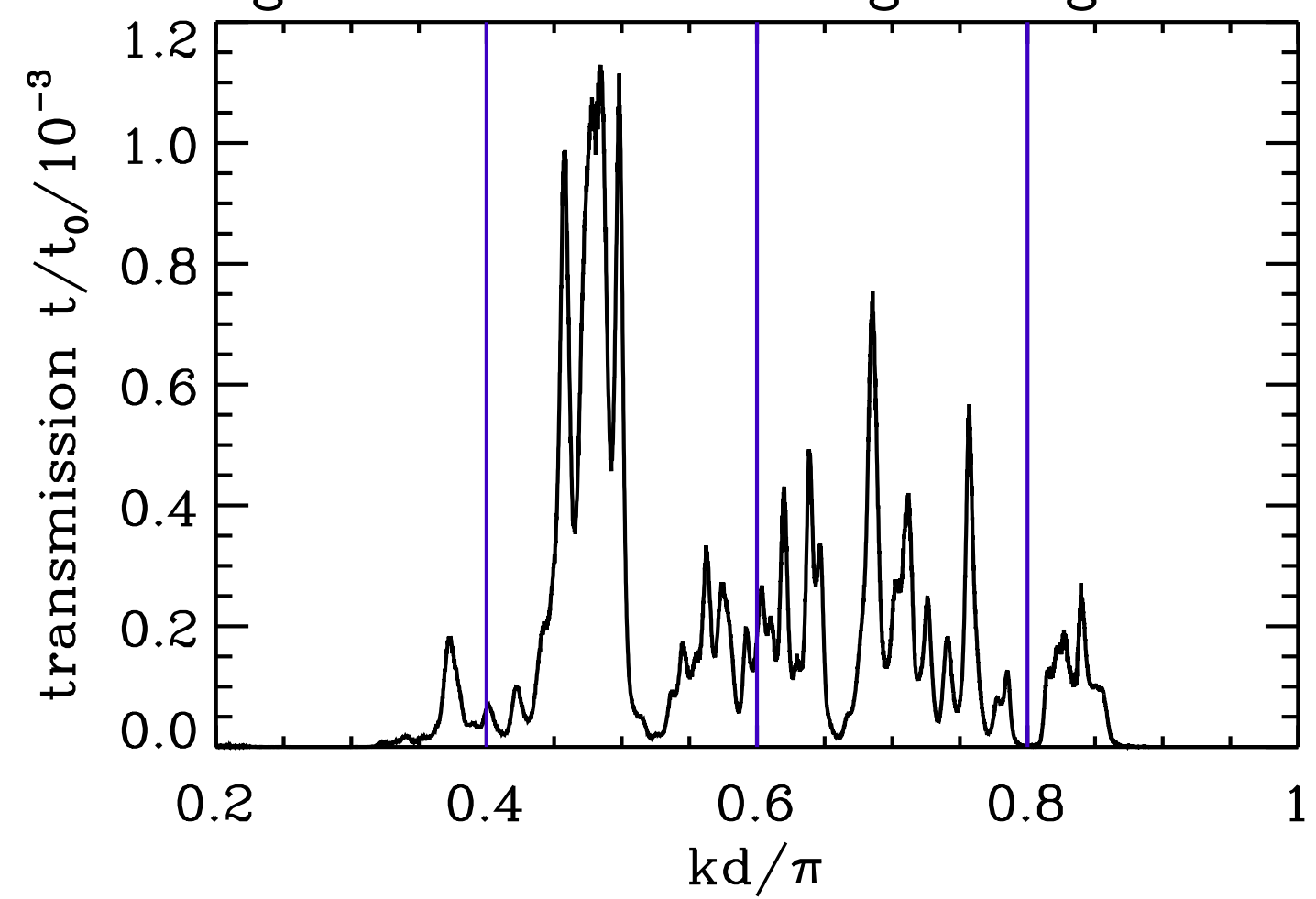

Micrometer screws are introduced randomly between 0 and $3 \mathrm{~mm}$. The transmission is roughly a percent of the transmission for the correlated disorder arrangements! 


\section{Correlated disorder}

\section{Enhanced localization}




\section{Transmission wave functions (random)}
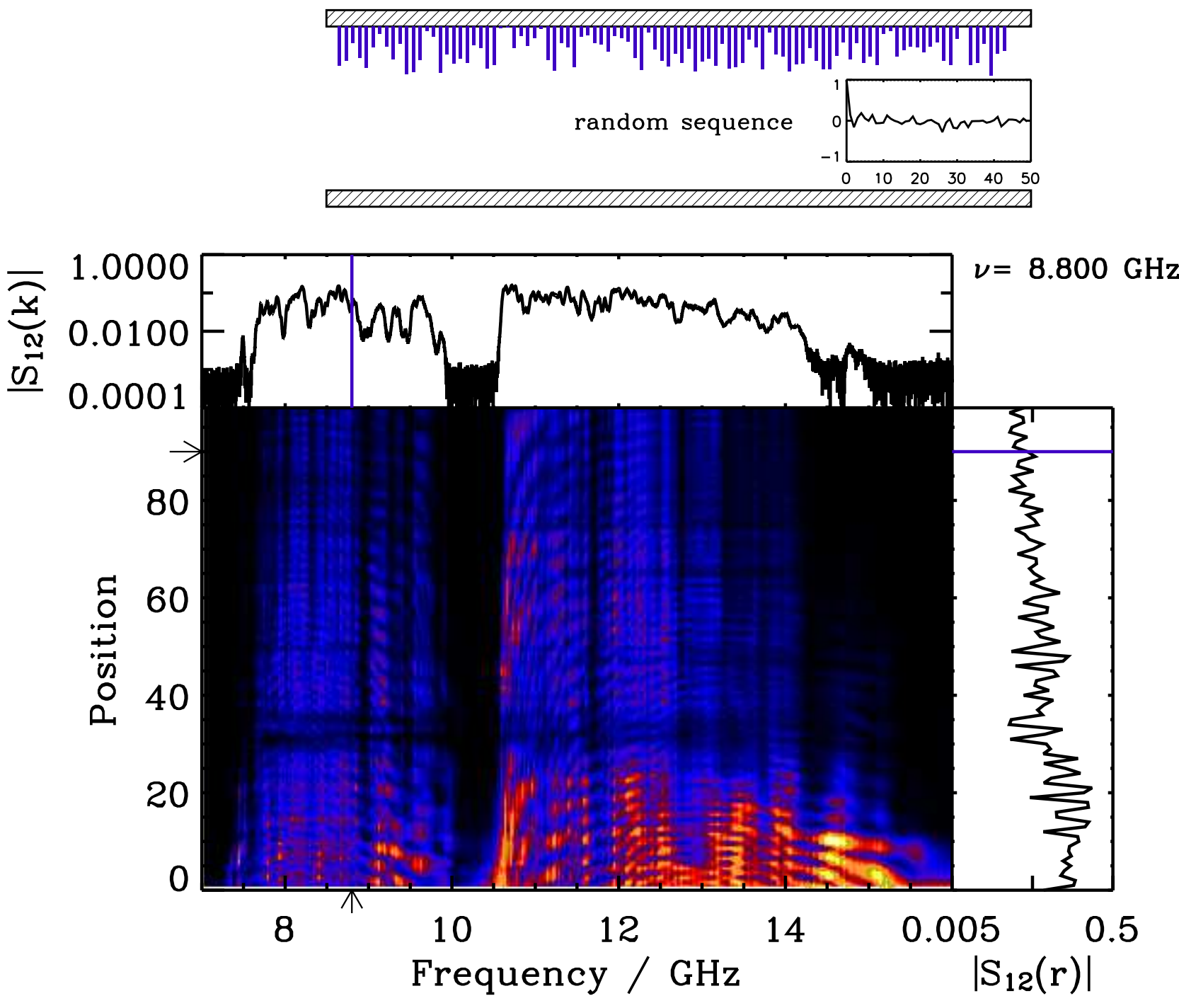


\section{Transmission wave functions (correlated)}
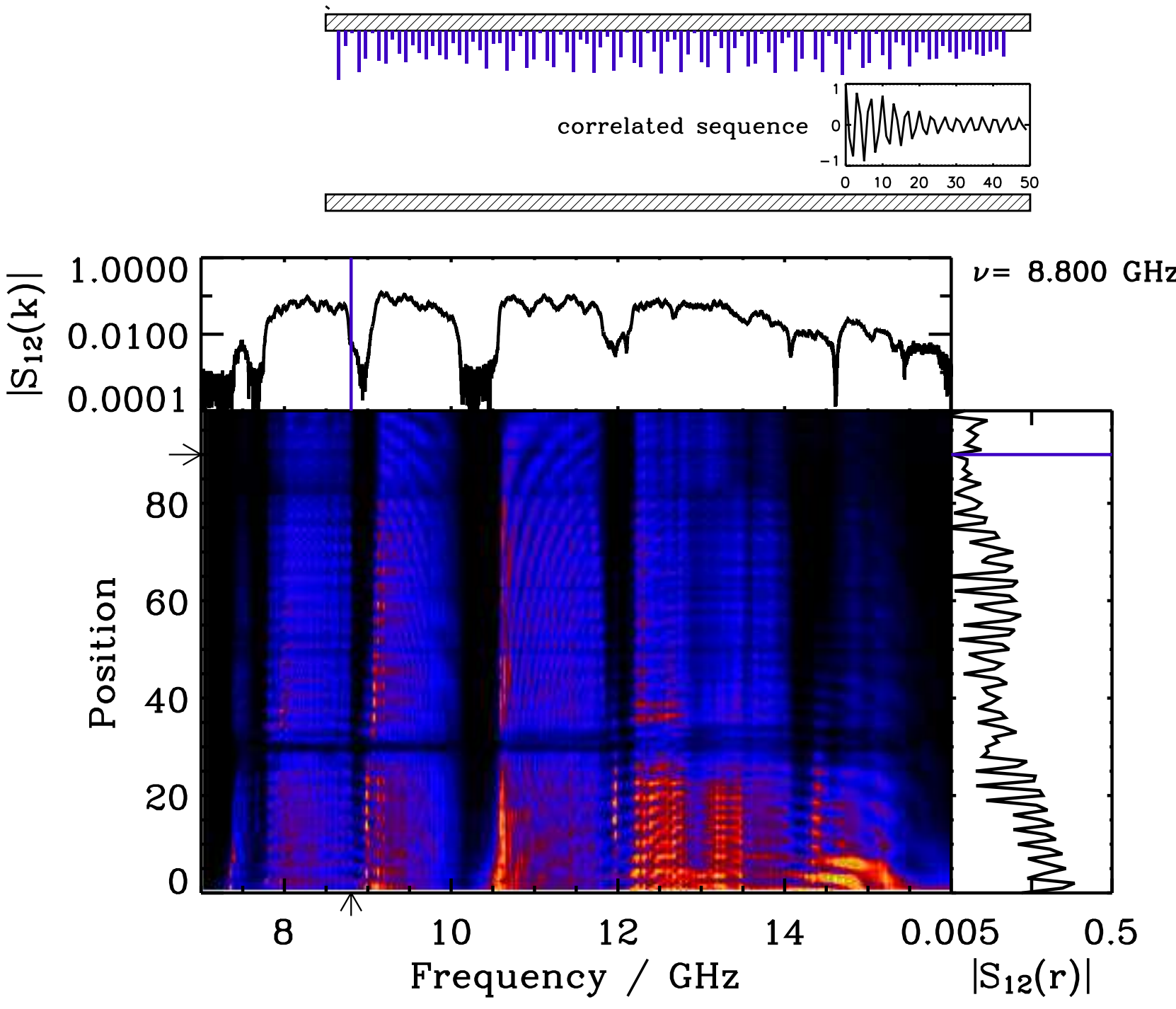


\section{Reflection wave functions (random)}

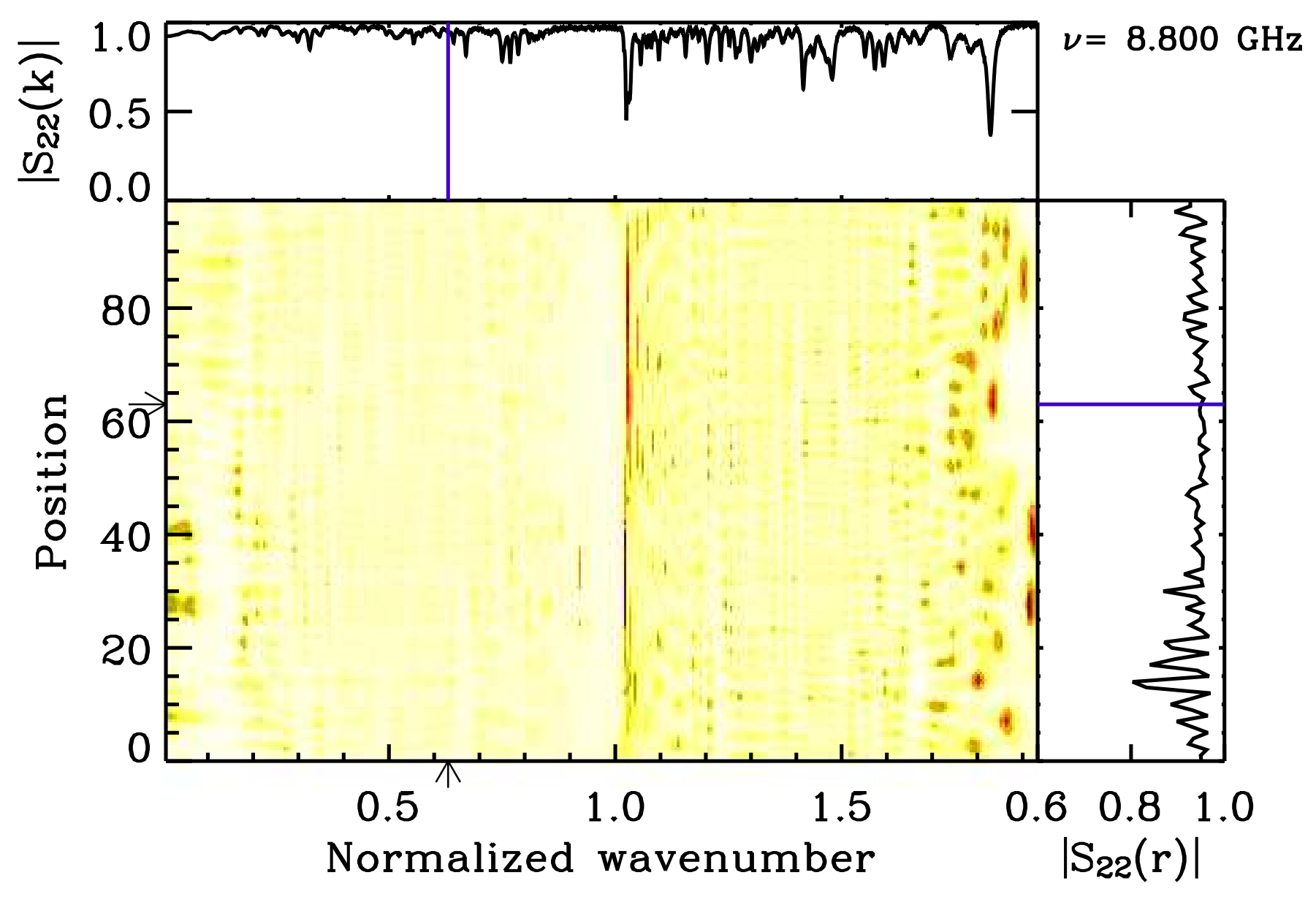




\section{Reflection wave functions (correlated)}

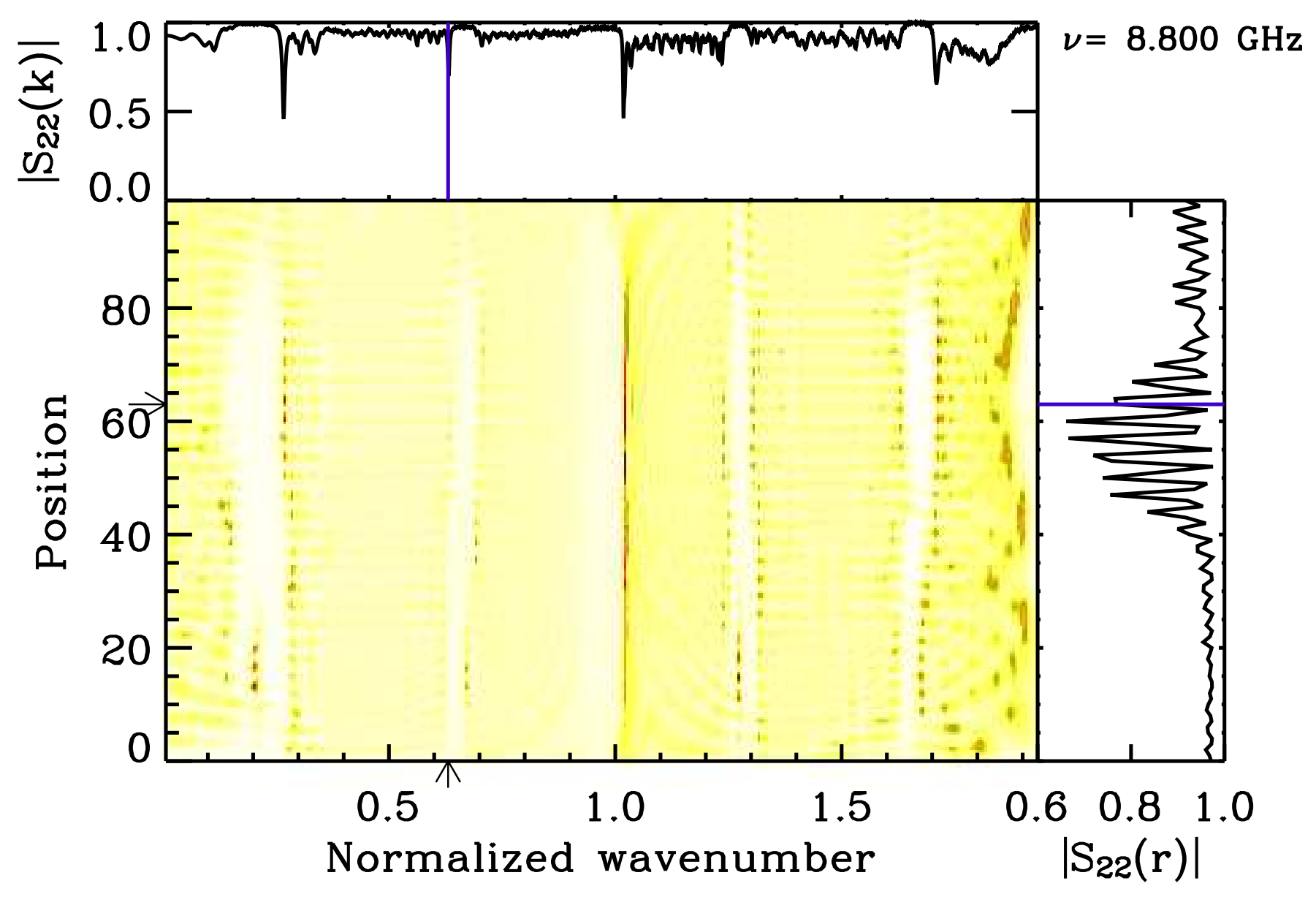




\section{Strongly localized wave functions}

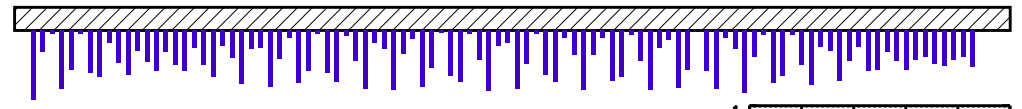

correlated sequence of Mhomm-

- transmission shows

band-gap structure

- seen in reflection as well

- localization within the gaps

- delocalization elsewhere

- reduced localization length by a factor of 15 (to $\approx 10$ )

[U.K., F. M. Izrailev, A. A. Krokhin, H.-J. Stöckmann. APL 2000]

[U.K., F. M. Izrailev, A. A. Krokhin, PRL 2008]
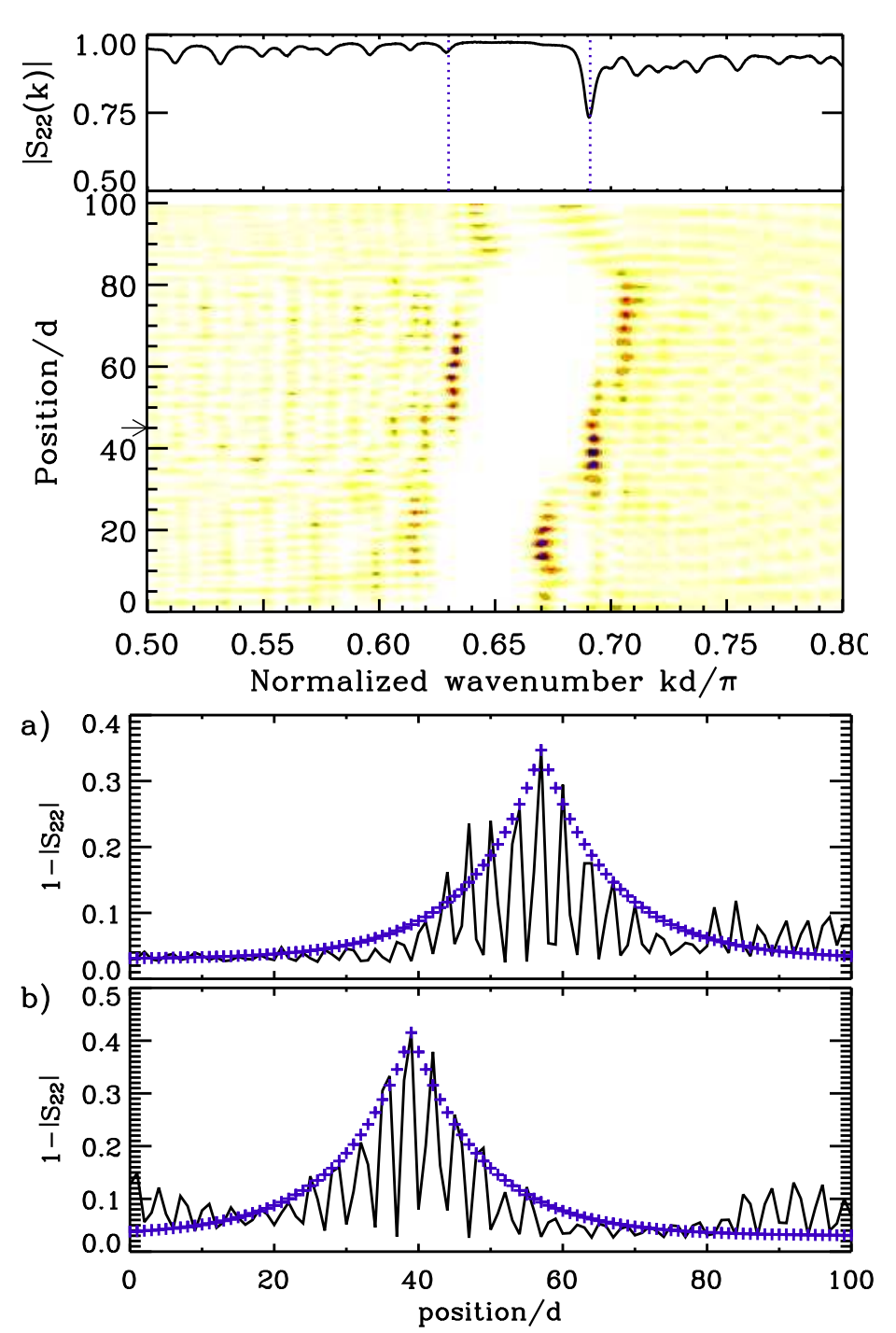


\section{Disorder}

\section{Fabry-Perot bands}




\section{Index variation (periodic lattice)}

Periodic lattice of teflon $(n \approx 1.4)$ pieces:

Single mode propagation within $7.5 \mathrm{GHz} \leq \nu \leq 15 \mathrm{GHz}$.
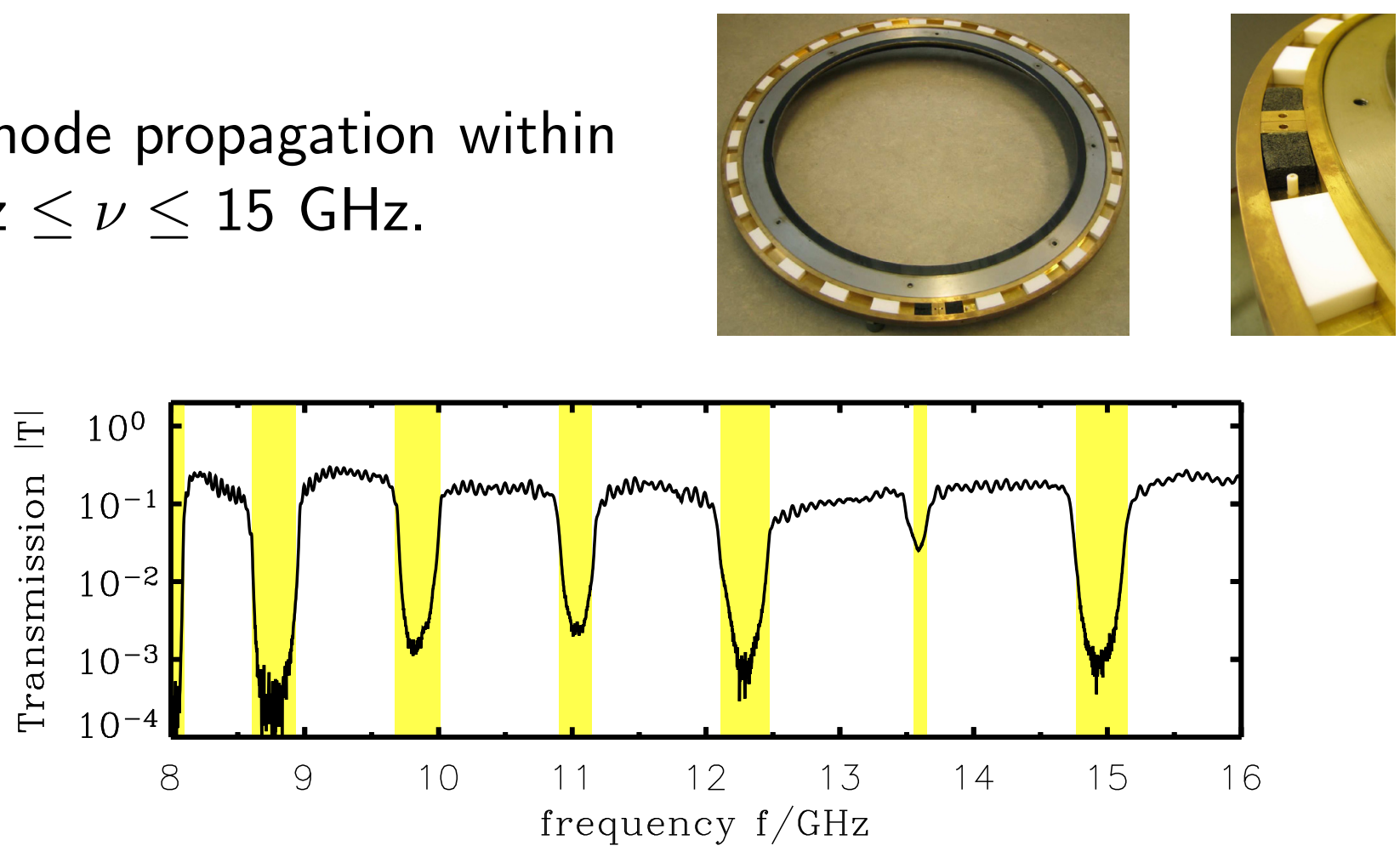

- periodic structures $\Rightarrow$ bands and gaps

¿ "photonic crystal" 


\section{Impurity}
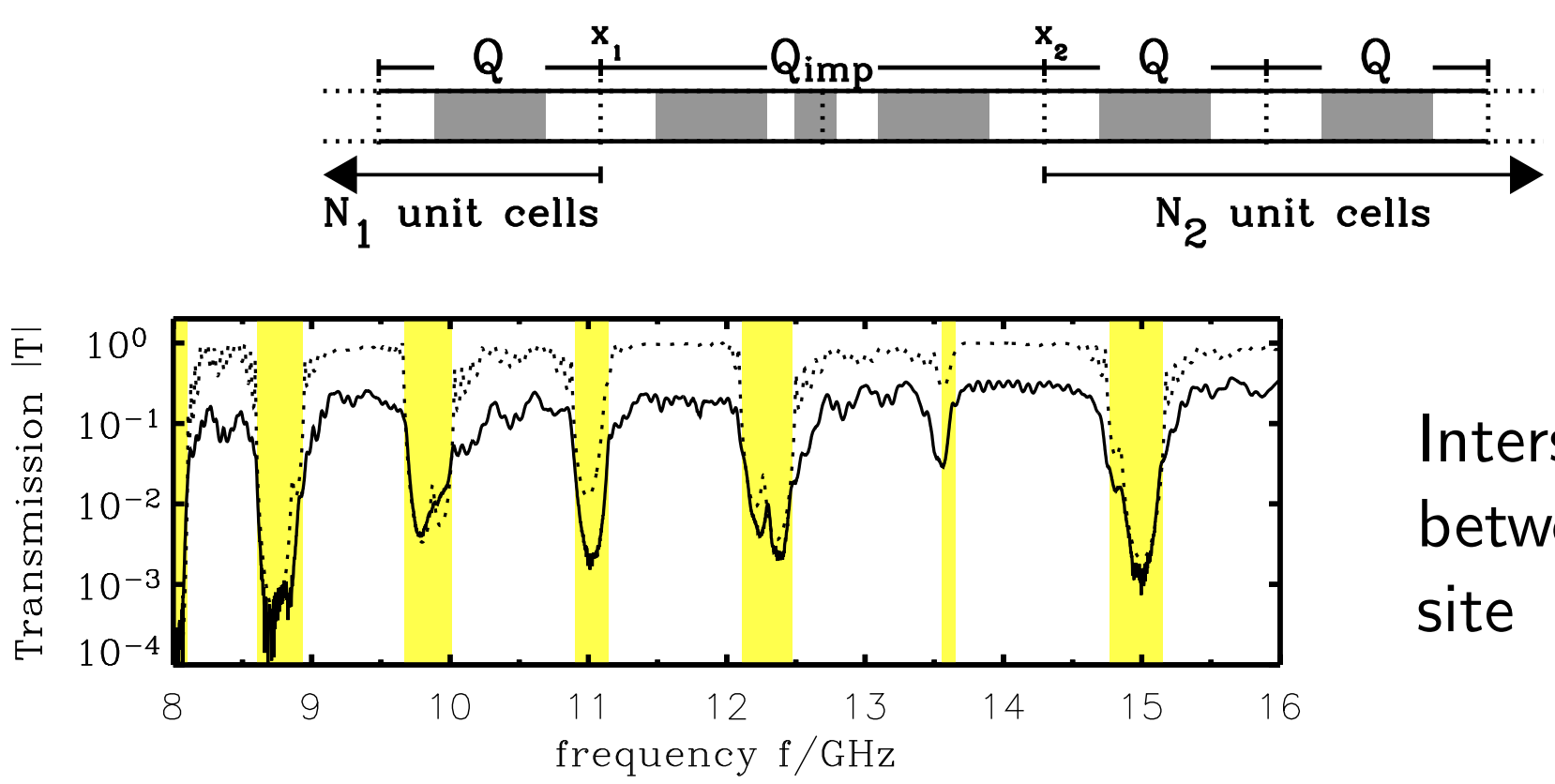

Interstitial impurity between 3rd and 4th site

ص impurities generate bound states in the gap

¿ impurity position $\Leftrightarrow$ band oscillations

¿ good agreement with transfer matrix simulations

- derived exact theoretical description

[G. Luna-Acosta, H. Schanze, U.K., H.-J. Stöckmann, New J. of Physics 10, 043005 (2008)] 


\section{Positional disorder with Fabry-Perot}

- No disorder in length of Teflon pieces

$\Rightarrow$ Perfect transmission at resonance frequency

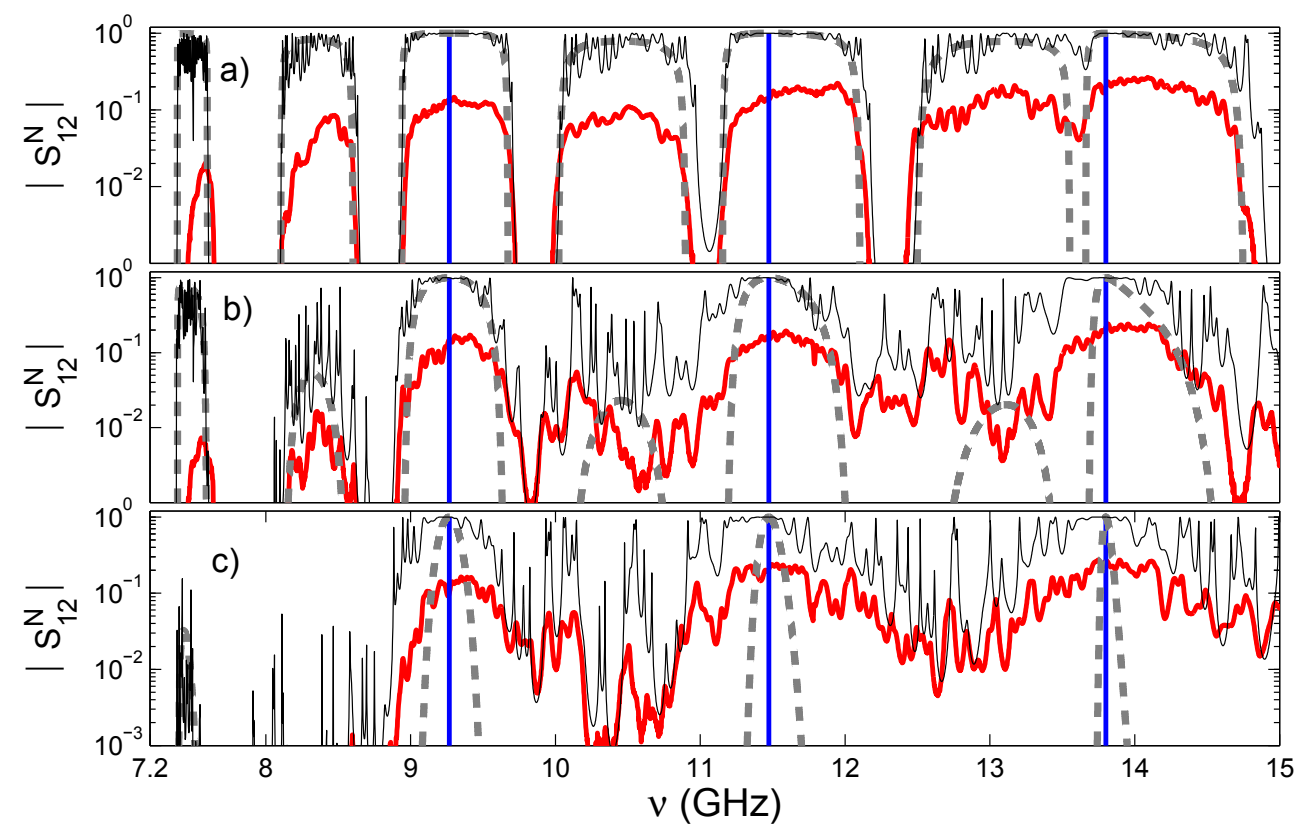

26-cells arrays:

Disorder:

(a) weak $\epsilon=3.0 \cdot 10^{-2}$

(b) medium $\epsilon=12.3$. $10^{-2}$

(c) strong $\epsilon=49.0 \cdot 10^{-2}$ black: numerics red: experiments Dashed: Analytics blue: teflon resonances

- two different band types

¿ behaviour on disorder strength different

- theoretical description for weak disorder

[G. A. Luna-Acosta, F. M. Izrailev, N. M. Makarov, U.K., and H.-J.Stöckmann, Phys. Rev. B 80, 115112 (2009)] 


\section{Quasi one dimensional Waveguides (Q1D)}

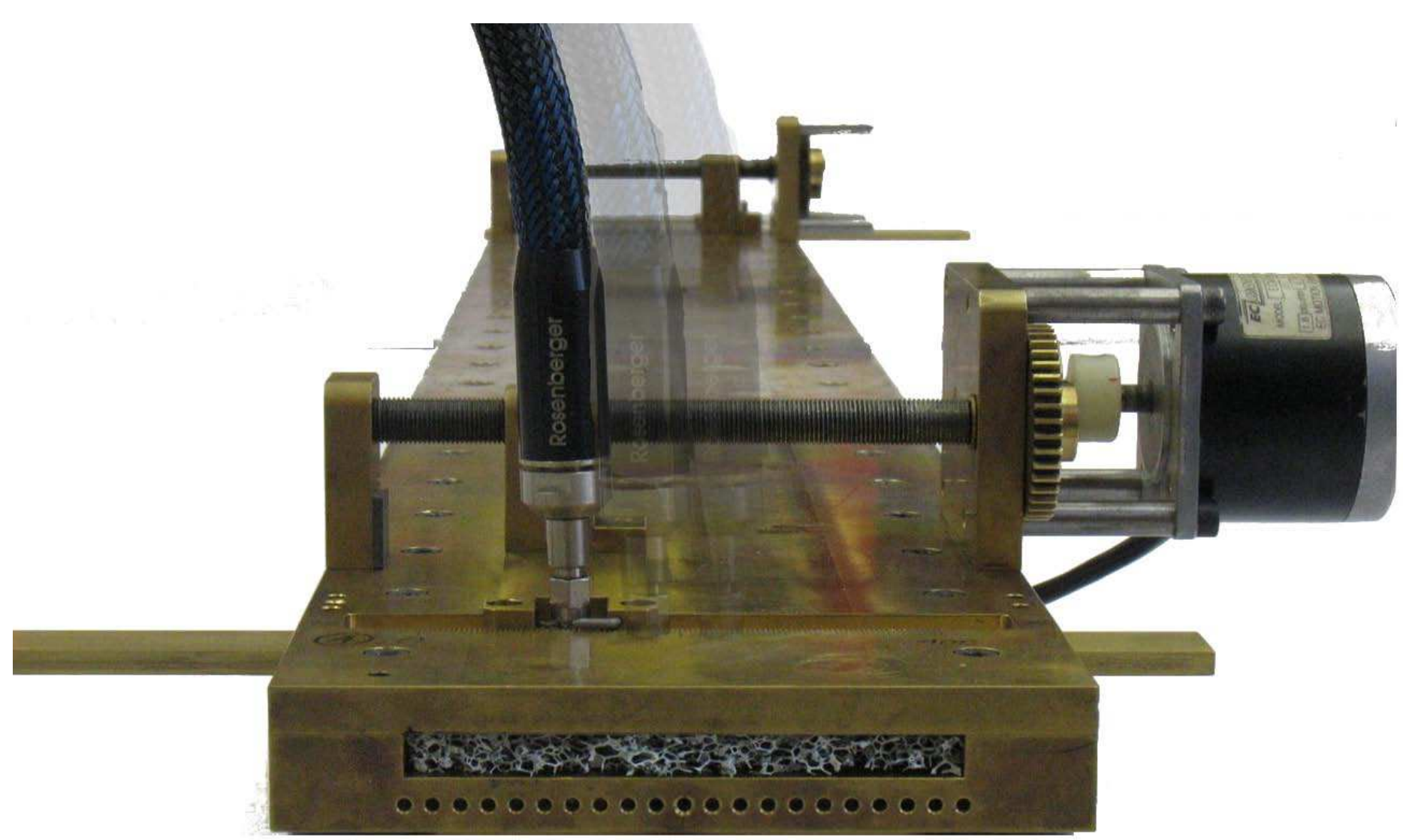




\section{Experimental set-up}

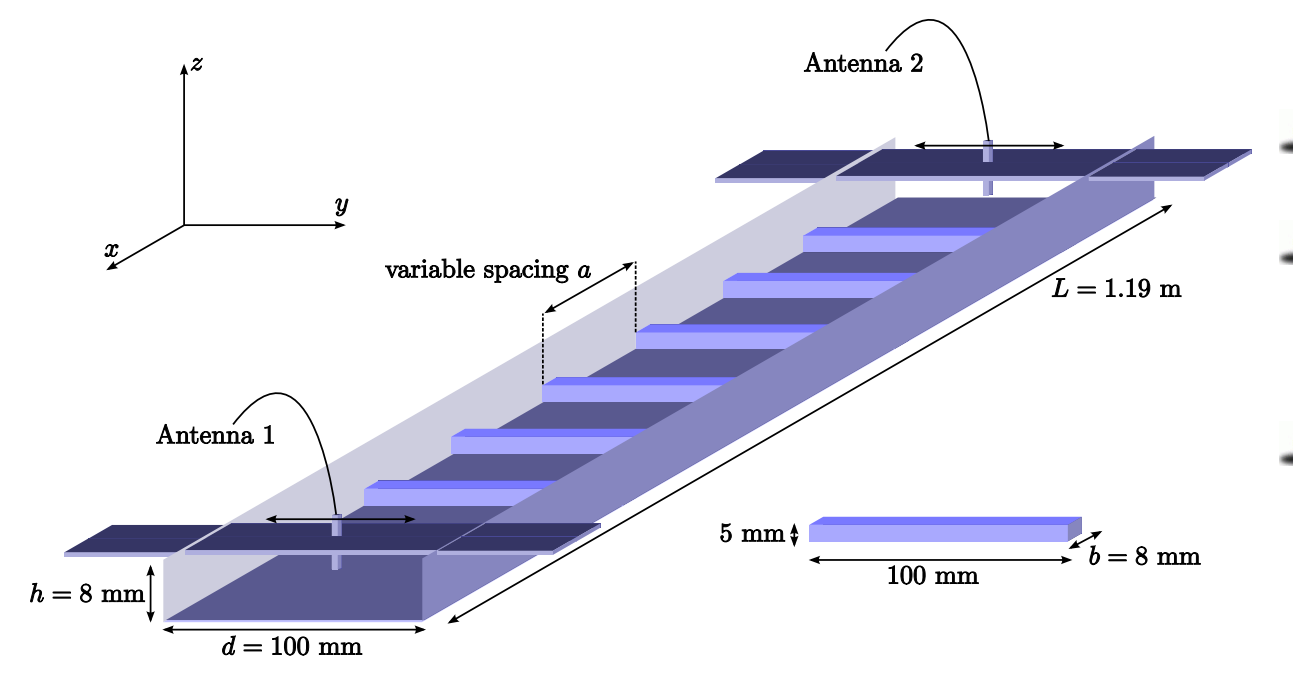

2 moveable antennas

scatterers here:

brass bars

- absorbers at both ends

mimic an infinite system 


\section{Experimental set-up}

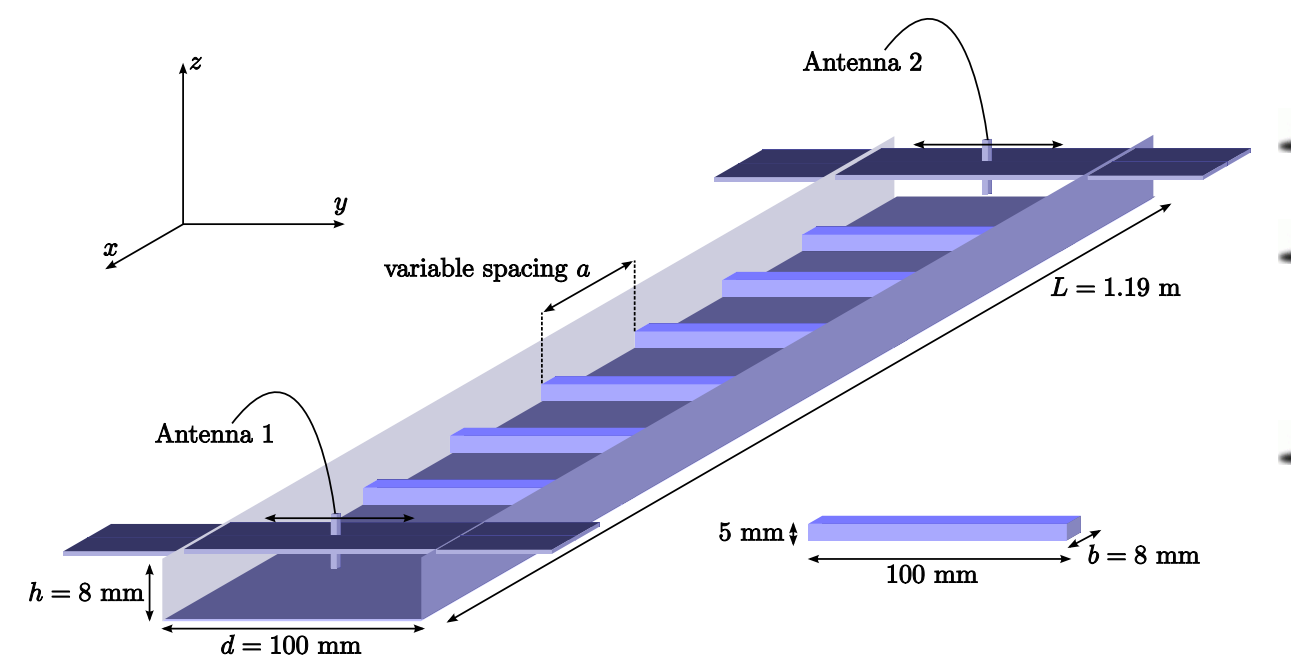

- 2 moveable antennas

¿ scatterers here:

brass bars

- absorbers at both ends

mimic an infinite system

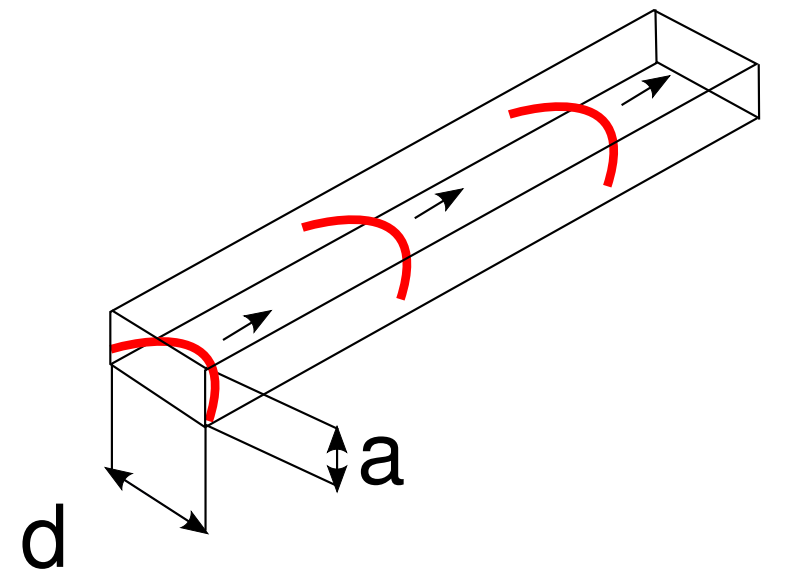

- measurement in position space

\ Fourier transform $\Rightarrow$ scattering matrix in mode representation

- wavenumber for each mode $n$ :

$k_{x, n}=\sqrt{k^{2}-\left(\frac{n \pi}{d}\right)^{2}}$ 


\section{Transmission (position representation)}

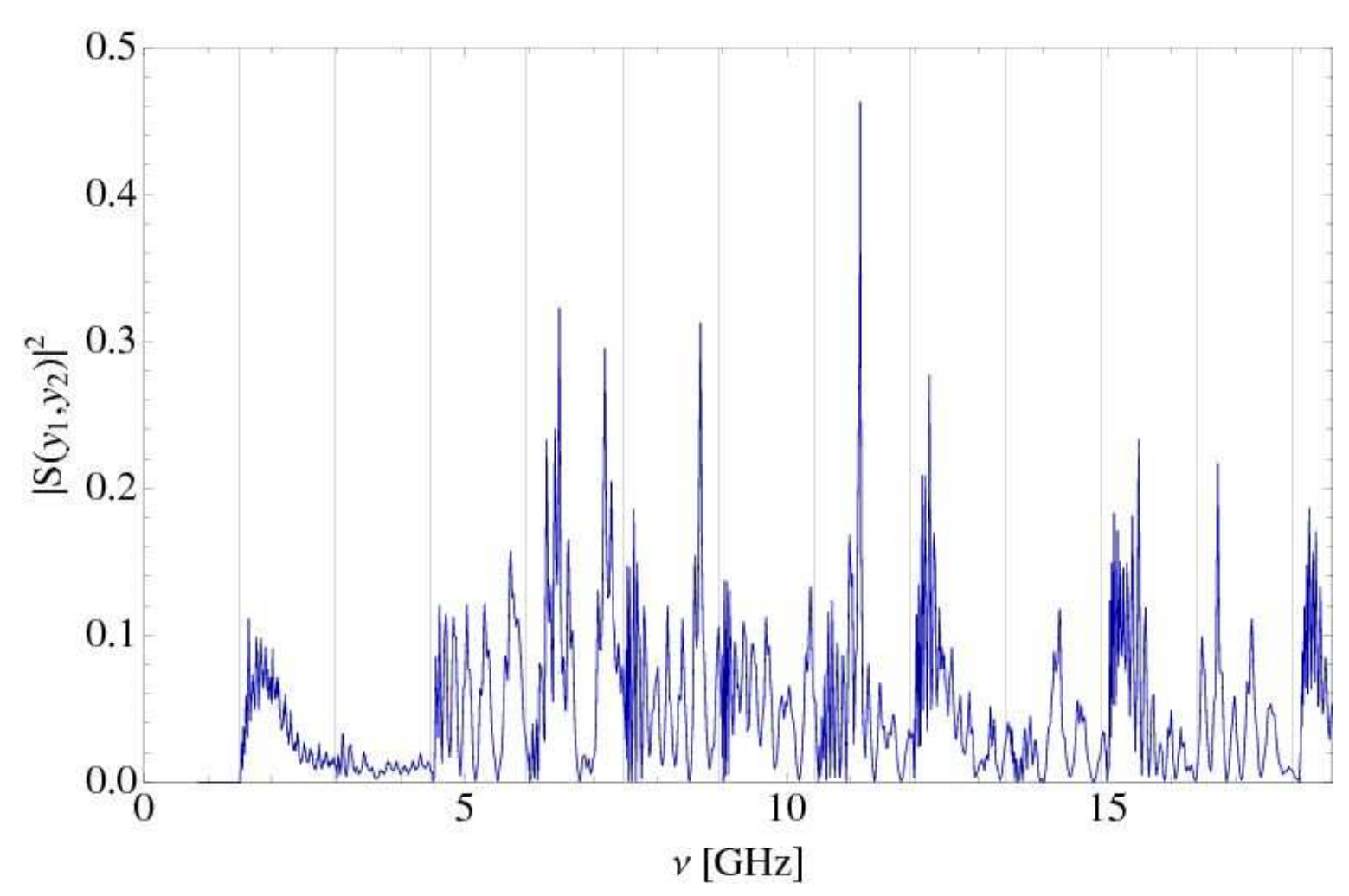

¿ fixed antenna positions $\left(y_{1}=y_{2}=55 \mathrm{~mm}\right)$

๑ vertical lines indicate mode openings

\ input data for Fouriertransform 


\section{Mode extraction}

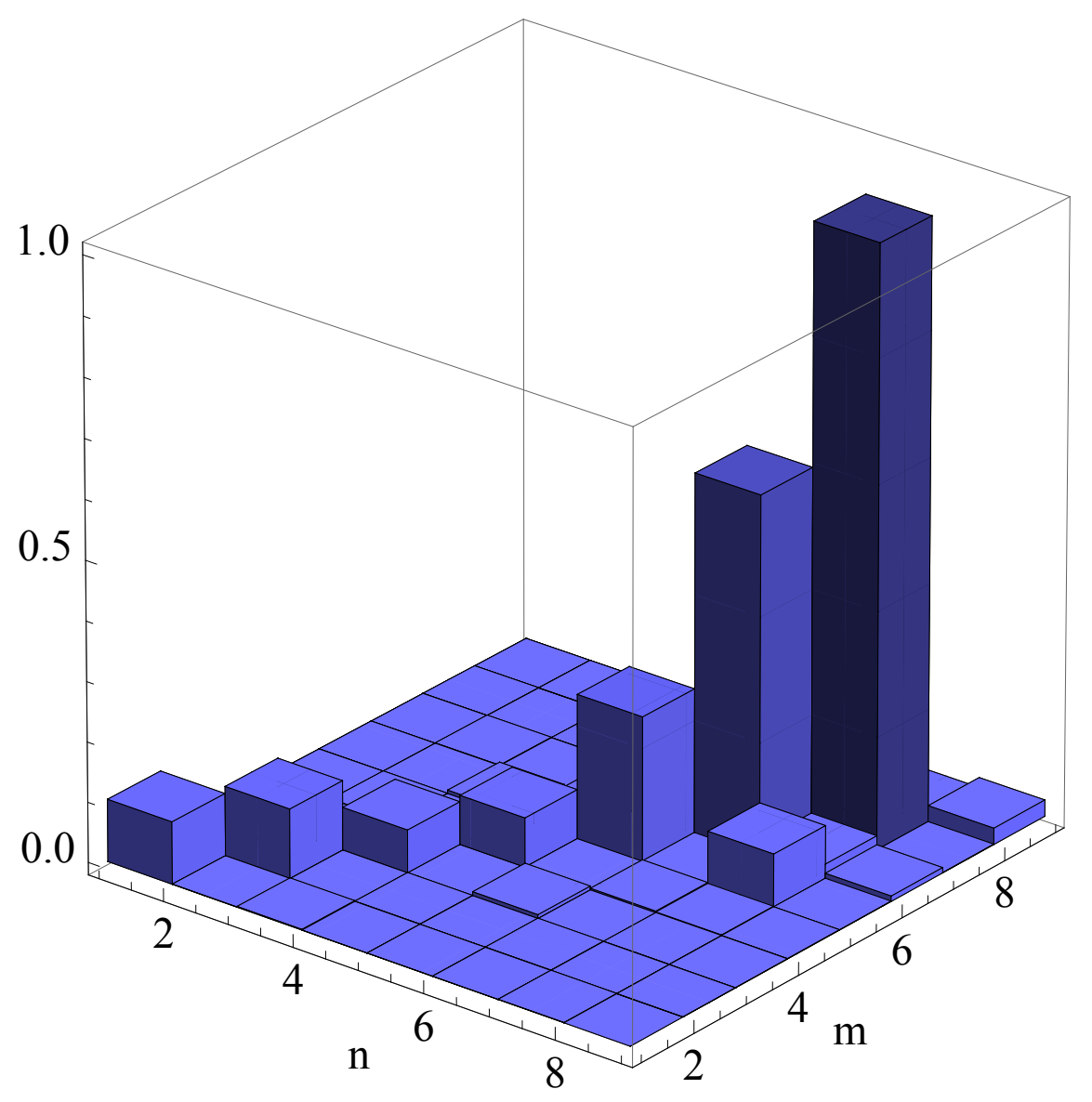

- modulus square of scattering matrix elements $\left|S_{n m}\right|$

๖ $\quad \nu=11 \mathrm{GHz}$ where 7 open modes exist 


\section{Transmission for 4th mode}

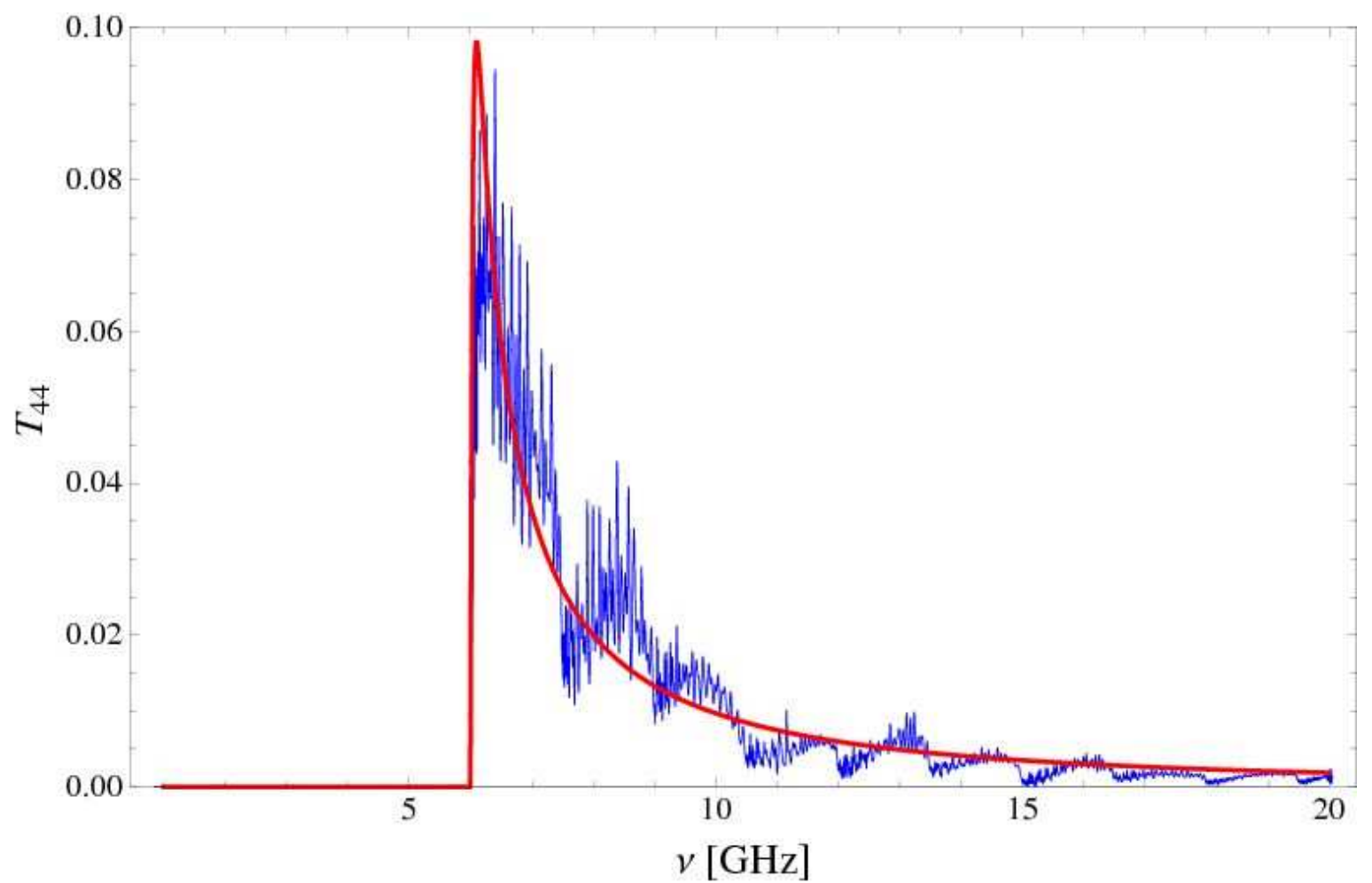

2 mode propagation for the 4th mode through of the empty waveguide (dark)

๖ $S_{n n} \sim G_{n}, \quad T_{n} \sim\left|G_{n}\right|^{2}$

$G_{n}:$ Green function for rectangular waveguide 


\section{Periodic structures}

Band structure example for 3rd (blue) and 4th mode (yellow)

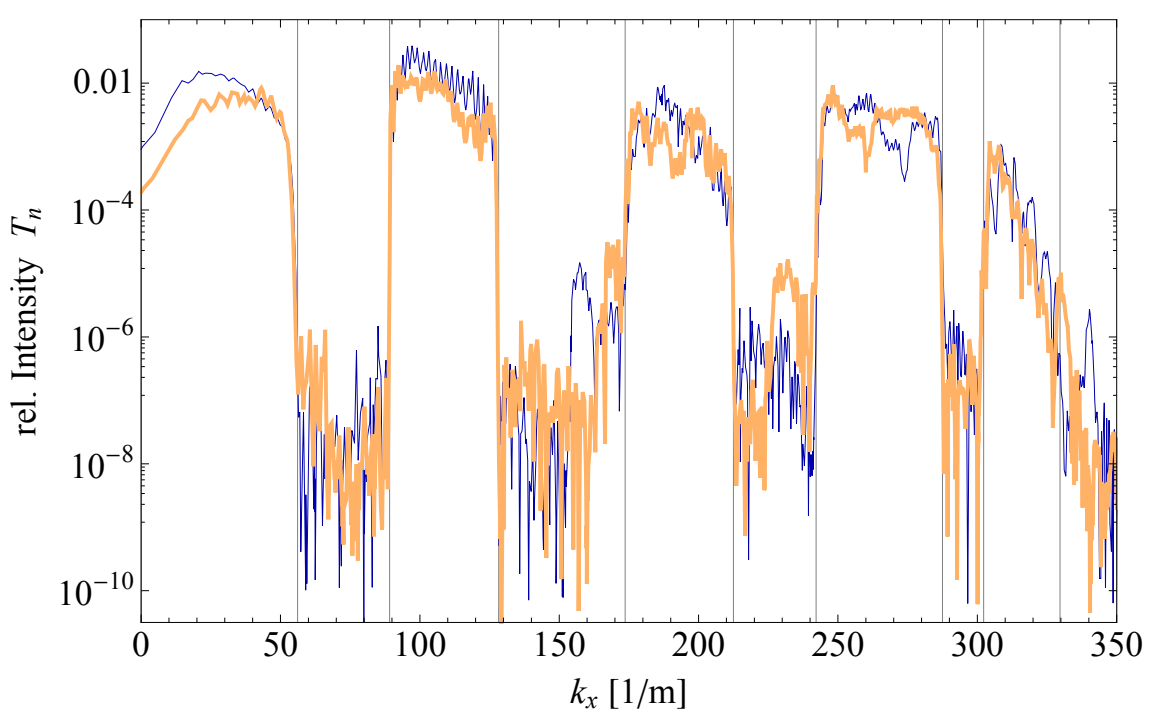

- clear band structure which is the same for all modes

- vertical lines indicate band edges

- description in terms of an effective bilayer model

- use band edges to determine the free parameters $\left(n_{a / b}, \alpha\right)$ from the dispersion relation: $\cos (\gamma)=\cos \left(k_{x} n_{a} a\right) \cos \left(k_{x} n_{b} b\right)-\alpha \cdot \sin \left(k_{x} n_{a} a\right) \sin \left(k_{x} n_{b} b\right)$ 


\section{Correlated disorder}

Band structure of 3rd mode:

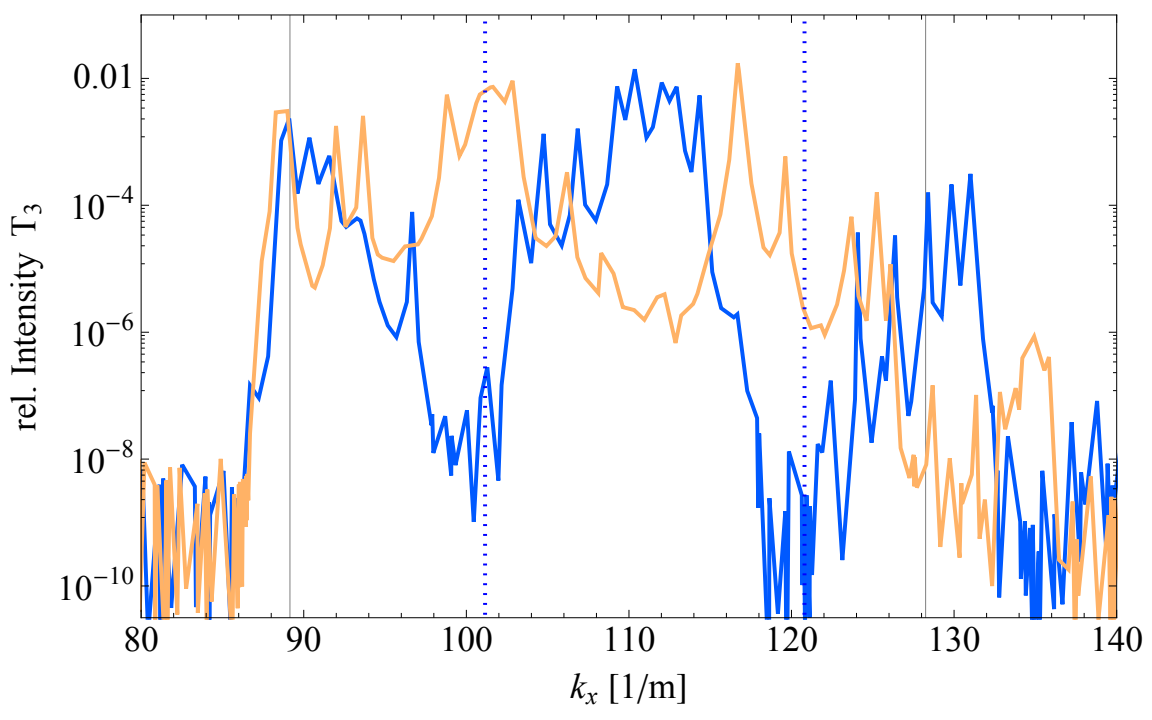

- transmission for white noise disorder in yellow

- transmission for correlated disorder in blue

\& blue dotted lines indicate the expected correlation gaps:

$\gamma / \pi=0.3$ and $\gamma / \pi=0.7$ 


\section{Fabry-Perot band}

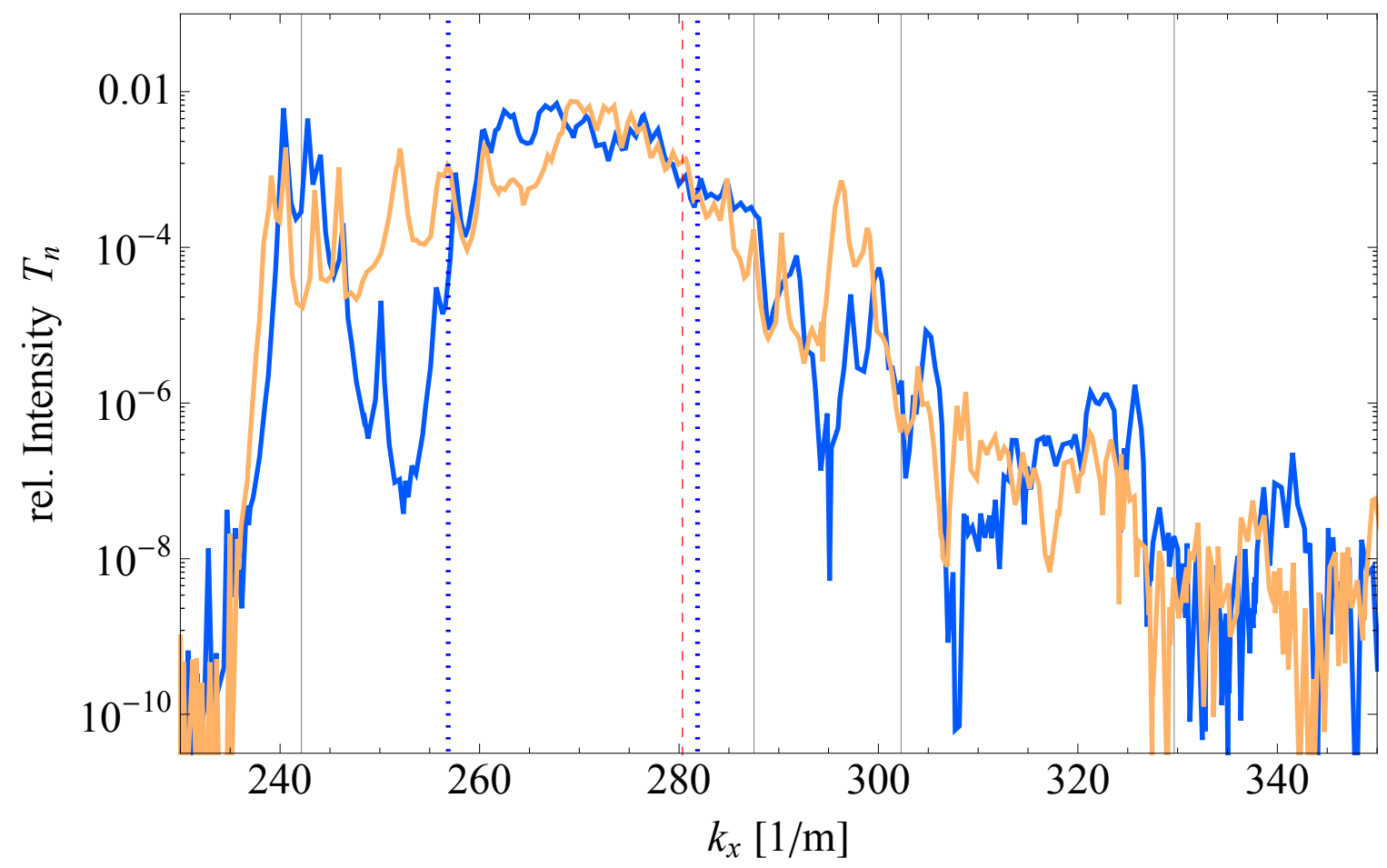

- width and height of metallic inset always the same $\Rightarrow$ Fabry-Perot band

¿ induced gap close to Fabry-Perot resonance disappeared

D other is shifted

[O. Dietz, U.K., H.-J. Stöckmann, F.M. Izrailev, N.M. Makarov, in preparation] 


\section{Summary}

- One dimensional waveguide

\& realization of Hofstadter butterfly

¿ induced band by correlated disorder

- enhanced localization by correlated disorder

\& Fabry-Perot bands

- Quasi 1D systems

¿ separation of modes

\& transport suppression in each modes by correlated disorder

\& interplay between Fabry-Perot band and correlations
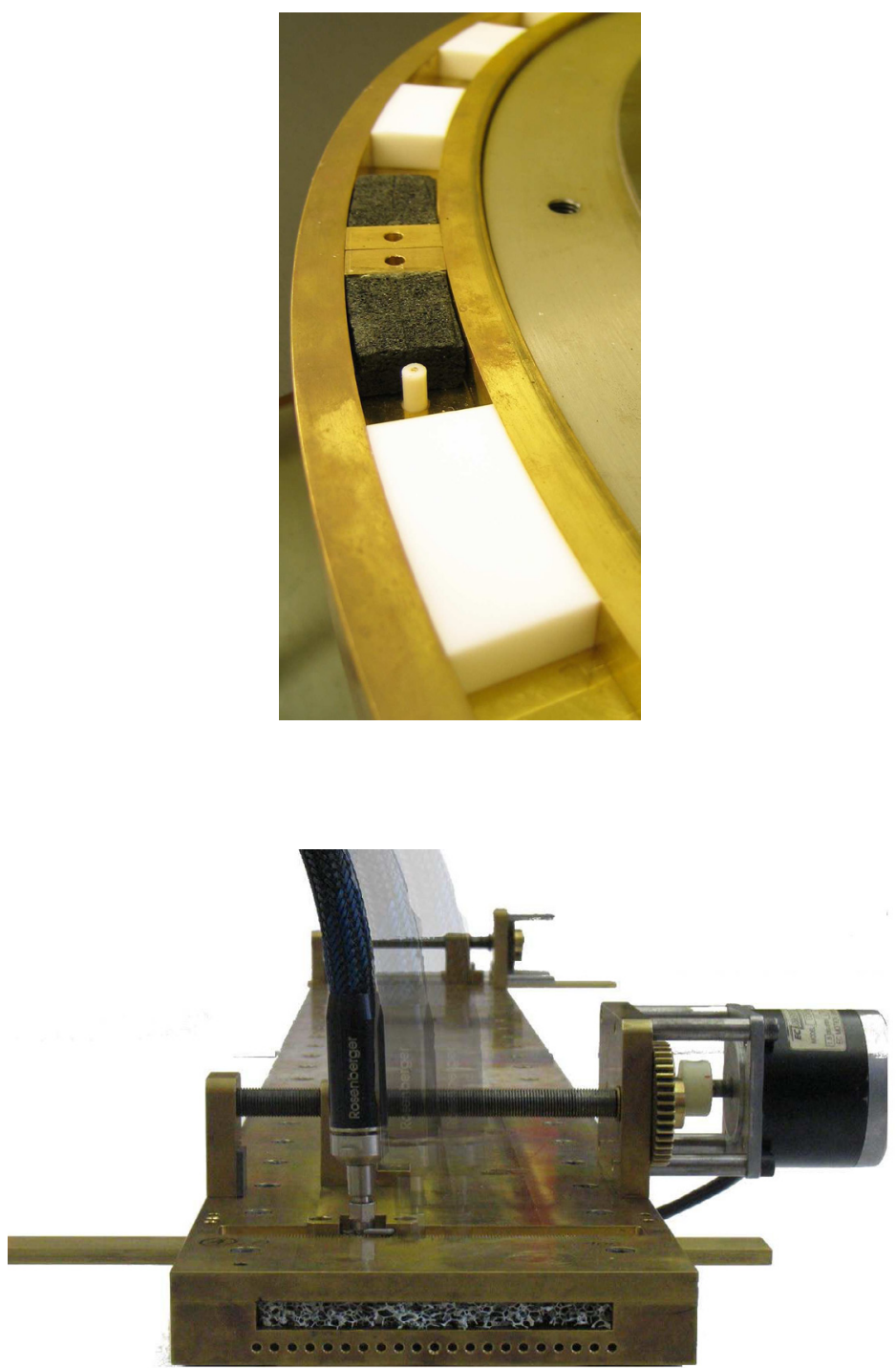


\section{Publications}

Publications that the presentations strongly depended on

¿ U. K., H.-J. Stöckmann. Phys. Rev. Lett. 80, 3232 (1998)

\& U.K., F. M. Izrailev, A. A. Krokhin, H.-J. Stöckmann. Appl. Phys. Lett. 77, 633 (2000)

¿ U.K., H.-J. Stöckmann. Physica E 9, 384 (2001)

¿ U. K., F. M. Izrailev, A. A. Krokhin, Phys. Rev. Lett. 100, $126402(2008)$

- G. A. Luna-Acosta, H. Schanze, U. K., H.-J. Stöckmann, New J. of Physics 10, 043005 (2008)

- G. A. Luna-Acosta, F. M. Izrailev, N. M. Makarov, U. K., H.-J.Stöckmann, Phys. Rev. B 80, 115112 (2009)

O O. Dietz, U. K., H.-J. Stöckmann, F. M. Izrailev, N. M. Makarov, in preparation (October 2010) 